ARTICLE

\title{
A macrophage-specific IncRNA regulates apoptosis and atherosclerosis by tethering HuR in the nucleus
}

Viorel Simion (1) 1,8, Haoyang Zhou ${ }^{1,2,8}$, Stefan Haemmig ${ }^{1}$, Jacob B. Pierce (1) ${ }^{1,3}$, Shanelle Mendes ${ }^{1}$, Yevgenia Tesmenitsky ${ }^{1}$, Daniel Pérez-Cremades (1D ${ }^{1}$, James F. Lee ${ }^{4}$, Alex F. Chen ${ }^{2}$, Nicoletta Ronda (1D) ${ }^{5}$, Bianca Papotti ${ }^{5}$, Jarrod A. Marto (id ${ }^{4,6,7} \&$ Mark W. Feinberg (i) ${ }^{1 \times}$

Long non-coding RNAs (IncRNAs) are emerging regulators of pathophysiological processes including atherosclerosis. Using RNA-seq profiling of the intima of lesions, here we identify a macrophage-specific IncRNA MAARS (Macrophage-Associated Atherosclerosis IncRNA Sequence). Aortic intima expression of MAARS increases by 270-fold with atherosclerotic progression and decreases with regression by $60 \%$. MAARS knockdown reduces atherosclerotic lesion formation by $52 \%$ in $\mathrm{LDLR}^{-/}-$mice, largely independent of effects on lipid profile and inflammation, but rather by decreasing macrophage apoptosis and increasing efferocytosis in the vessel wall. MAARS interacts with HuR/ELAVL1, an RNA-binding protein and important regulator of apoptosis. Overexpression and knockdown studies verified MAARS as a critical regulator of macrophage apoptosis and efferocytosis in vitro, in an HuRdependent manner. Mechanistically, MAARS knockdown alters HuR cytosolic shuttling, regulating HuR targets such as p53, p27, Caspase-9, and BCL2. These findings establish a mechanism by which a macrophage-specific IncRNA interacting with HuR regulates apoptosis, with implications for a broad range of vascular disease states.

\footnotetext{
${ }^{1}$ Department of Medicine, Cardiovascular Division, Brigham and Women's Hospital, Harvard Medical School, Boston, MA, USA. ${ }^{2}$ Department of Cardiology, The Third Xiangya Hospital of Central South University, Changsha, Hunan, China. ${ }^{3}$ Feinberg School of Medicine, Northwestern University, Chicago, IL, USA. ${ }^{4}$ The Blais Proteomics Center, Dana-Farber Cancer Institute, Boston, MA, USA. ${ }^{5}$ Department of Food and Drug, University of Parma, Parma, Italy.

${ }^{6}$ Departments of Cancer Biology and Oncologic Pathology, Dana-Farber Cancer Institute, Boston, MA, USA. 7 Department of Pathology, Brigham and Women's Hospital, Harvard Medical School, Boston, MA, USA. ${ }^{8}$ These authors contributed equally: Viorel Simion, Haoyang Zhou. ${ }^{凶}$ email: mfeinberg@bwh.harvard.edu
} 
therosclerosis is one of the leading causes of death and disability worldwide. It is a complex disease characterized by lipid accumulation within the arterial wall, inflammation, and apoptosis. Innate immune cells, in particular macrophages, play a pivotal role in atherosclerosis initiation and progression $^{1,2}$. A growing number of studies demonstrate that advanced plaques contain a higher proportion of apoptotic cells than early plaques ${ }^{3,4}$. Accrual of modified LDL can induce macrophage or vascular smooth muscle cell apoptosis, which in earlier plaques are cleared via a process termed efferocytosis ${ }^{3}$. However, as macrophages are triggered into apoptosis, efferocytosis is rendered defective, an effect leading to plaque necrosis and adverse remodeling of the plaque architecture predisposing to vulnerable plaques ${ }^{5,6}$. As such, macrophage apoptosis is a critical contributor to atherosclerosis and plaque necrosis. However, significant gaps remain in the molecular underpinnings that regulate macrophage apoptosis in advanced atherosclerotic plaques.

The recent recognition that $<2 \%$ of the human genome encodes proteins has opened further opportunities to better understand regulatory pathways in vascular health and disease $\mathrm{e}^{7,8}$. The majority of biologically active RNAs that cannot be translated into proteins are long non-coding RNAs (lncRNAs) measuring more than 200 nucleotides in length and display mRNA-like characteristics such as being $5^{\prime}$-capped, spliced, and polyadenylated. There is increasing recognition that lncRNAs serve as potent regulators of key cellular processes mediated by their ability to interact with RNA, DNA, proteins, or RNA-binding proteins ${ }^{9,10}$. While there are more lncRNA transcripts than protein-coding genes, their expression, function, and mechanistic roles are poorly defined in atherosclerosis or relevant cell types found in the progression of early or late plaques. Accumulating studies demonstrate that lncRNAs are often enriched in a tissue- or cell-specific manner and regulate significant phenotypic effects ${ }^{11}$, while their subcellular localization pattern can provide additional insights into their mechanistic roles ${ }^{10}$. Discovery of $\operatorname{lncRNAs}$ specifically expressed in the intima of atherosclerotic lesions during the progression and regression phases may provide further insights for their roles in atherosclerosis, and potentially decipher mechanisms for apoptosis of advanced lesions.

The RNA-binding protein, HuR (also known as ELAV1) typically binds to AU-rich elements (ARE) to mediate transcript stability, typically of mRNA translation ${ }^{12,13}$. HuR regulates genes involved in key cellular processes such as apoptosis, hypoxia, tumorigenesis, among others ${ }^{14-16}$. HuR function is often linked to its cellular translocation from the nucleus to the cytoplasm ${ }^{16}$. However, a functional role of a specific lncRNA in HuR cytoplasmic shuttling has not been described.

In this study, we have identified and characterized the lncRNA Macrophage-Associated Atherosclerosis lncRNA Sequence $(M A A R S)$ as an essential regulator of macrophage apoptosis, efferocytosis, and plaque necrosis in atherosclerosis of $\mathrm{LDLR}^{-1-}$ mice by direct interaction with the RNA-binding protein $\mathrm{HuR}$, a key mediator of cellular apoptosis. These findings may provide insights into the pathophysiology of a broad range of chronic disease states associated with maladaptive macrophage apoptosis.

\section{Results}

Discovery of MAARS from the intima of atherosclerotic lesions. To discover the presence of lncRNAs in the aortic intima during the progression and regression phases of atherosclerosis, RNA was derived from the aortic intima of $\mathrm{LDLR}^{-1-}$ mice after a progression phase of 0,2 , and 12 weeks on high cholesterol diet (HCD) (groups $1-3)$ and from a regression phase at 18 weeks after 6 weeks of resumption to a normal chow diet (group 4) (Fig. 1a). The aortic intima was used for RNA-Seq profiling to capture differentially expressed lncRNAs (log2-fold change $(1.5)$; FDR $<0.05)$ and analyzed using DeSeq 2 and NOR algorithms as previously described ${ }^{17}$. Both algorithms identified 14 lncRNAs that were commonly dysregulated (Fig. 1b, c). The top differentially regulated transcript was the lncRNA Gm14461 with a 270-fold increase in expression in the aortic intima after 12 weeks of HCD during the progression phase (group 3), which decreased in expression by $60 \%$ during lesion regression (group 4), as verified by RT-qPCR (Fig. 1b and Supplementary Fig. 1a). The presence of macrophages and expression of this lncRNA in the aortic intima was further verified by RTqPCR (Supplementary Fig. 1b, c).

MAARS characterization as a macrophage-specific lncRNA. Gm14461 expression is highly expressed in bone marrowdifferentiated macrophages (BMDMs) and the macrophage cell line RAW264.7 (Fig. 1d). In contrast, the Gm14461 transcript was undetectable in all the other cells types tested, including endothelial cells (primary isolated endothelial cells from liver and lung, and the endothelial cell line Bend.3), vascular smooth muscle cells (primary mouse aortic smooth muscle cells and the MOVAS cell line), NIH3T3 fibroblasts, or freshly isolated bone marrow or peripheral blood mononuclear cells (PBMCs) (Fig. 1d). While Gm14461 transcript is undetectable in mouse bone marrow, its expression increases exponentially up to 283 -fold after 7 days of differentiation in the presence of macrophage colony stimulating factor (MCSF) (Fig. 1e). Similar trends were observed in macrophages differentiated from PBMCs and spleen cells (Supplementary Fig. 1d). For this reason, we named this lncRNA MAARS. MAARS was detected only in the spleens of C57BL/6 mice, where a high number of macrophages are located, whereas it was undetectable in all other organs (Fig. 1f). Moreover, we have isolated spleens and sorted different cell populations using magnetic beads labeled with specific antibodies such as F4/80 (macrophage marker), CD11c (dendritic cells and macrophages), CD8a (T cells), CD19 (B cells), and negative sorting of B cells, and found that MAARS was highly enriched in the F4/80-sorted fraction (Fig. 1g). In contrast, the highest expression of MAARS was detected in the intima of $\mathrm{LDLR}^{-1-}$ mice fed HCD for 12 weeks (12-fold higher than the spleen, Fig. 1h), in accordance with the abundance found in our initial RNA-seq screening data (Fig. 1b, h). To further assess the regulation of MAARS expression specifically in the macrophages with different phases of atherosclerosis (Fig. 1b, c), FACS-sorted F4/80+/CD11b ${ }^{+}$macrophages were isolated during atherosclerosis progression and regression. $M A A R S$ expression was eightfold increased in the $\mathrm{F} 4 / 80^{+} / \mathrm{CD} 11 \mathrm{~b}^{+}$ double-positive population of macrophages compared to the negative-stained cells in the progression group, while in the atherosclerosis regression group MAARS expression decreased by $46 \%$ in $\mathrm{F} 4 / 80^{+} / \mathrm{CD} 11 \mathrm{~b}^{+}$macrophages (Fig. 1i). To obtain additional verification for its macrophage specificity, MAARS (nuclear staining) was visualized by RNA-in situ hybridization (ISH) (red) in close proximity to the cytoplasmic macrophage marker Mac-2 (antibody-stained in green) in the aortic sinus (Fig. $1 \mathrm{j}$ and Supplementary Fig. 1e).

In 5'RACE PCR studies, we confirmed the six exons currently annotated for MAARS in the NCBI and Ensemble databases, in accordance with RNA-seq data (Supplementary Fig. 1f). Intriguingly, MAARS is annotated as a lncRNA in the Ensembl database, while in NCBI it is annotated as an mRNA. Hence, we sought to elucidate this transcript by first checking the coding probability using the in silico coding potential assessment tool (CPAT) and found that MAARS has a similar score to other welldescribed lncRNAs such as HOTAIR, NEAT1, or SMILR (Fig. 1k). Consequently, for in vitro validation of peptide coding 
a
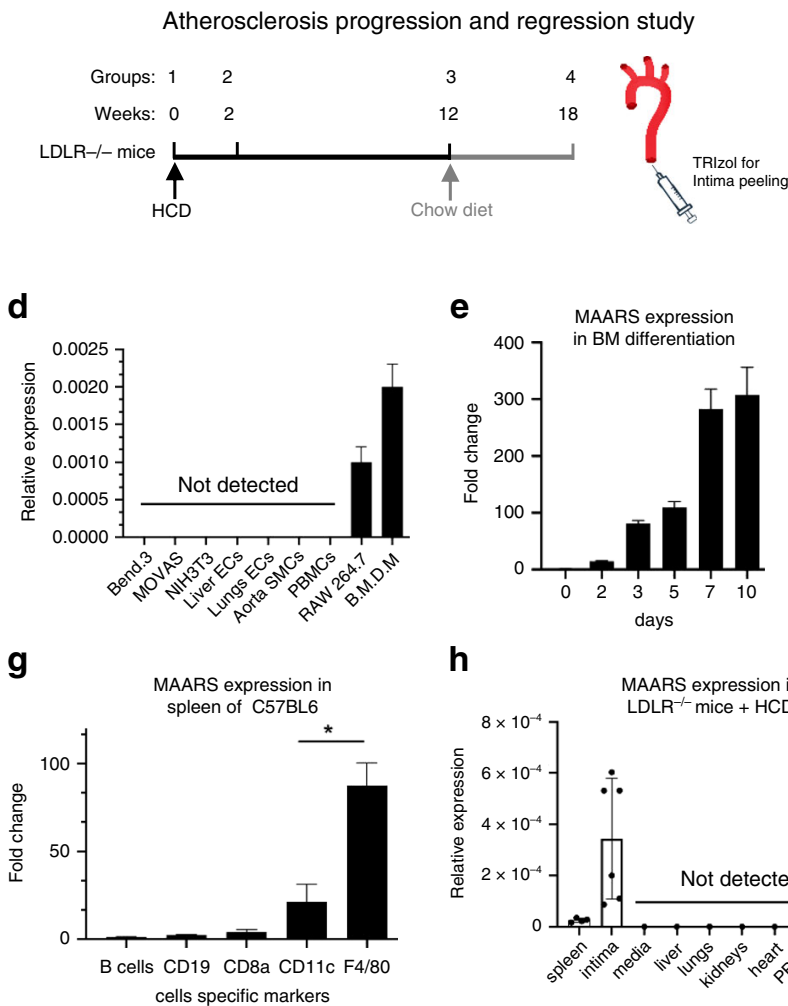

h
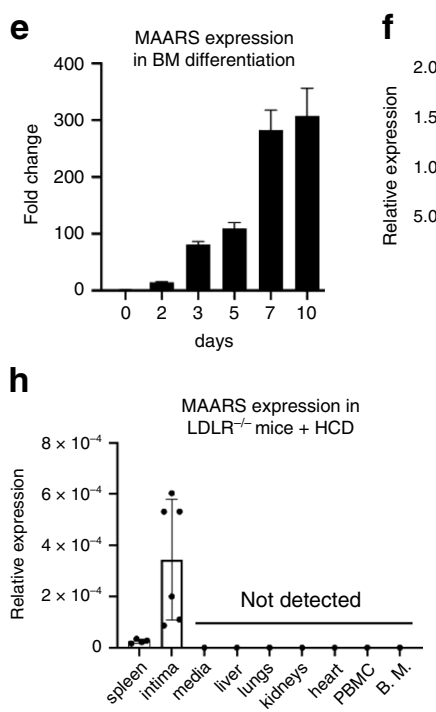

b

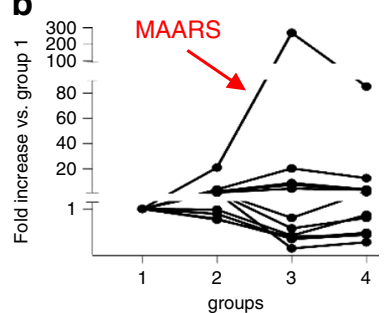

f
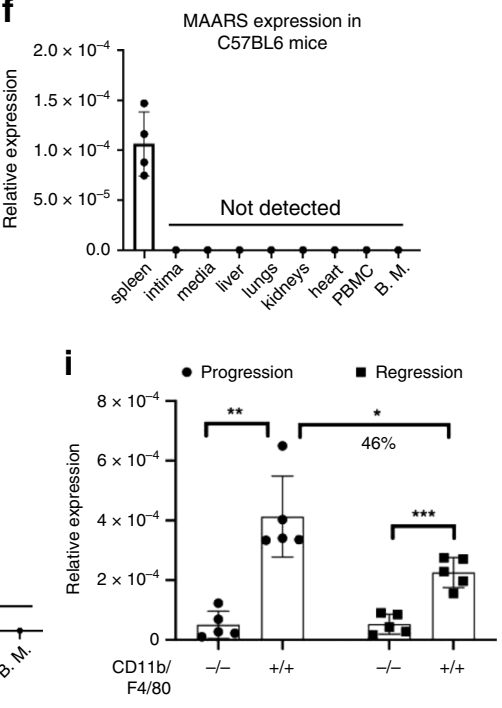

C

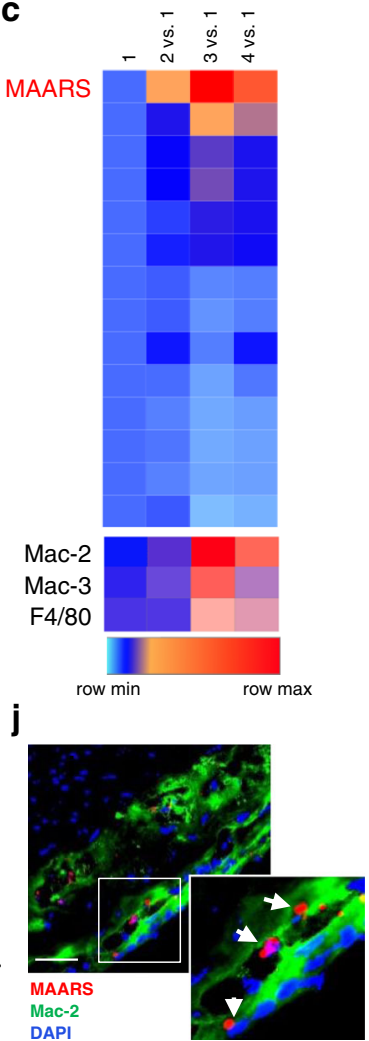

$\mathbf{k}$

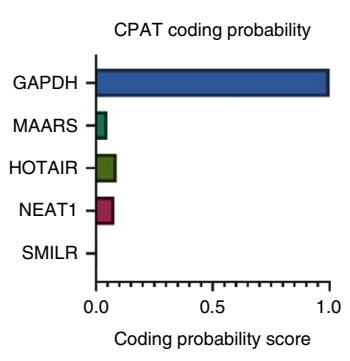

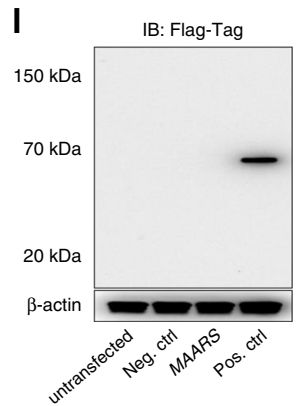

m

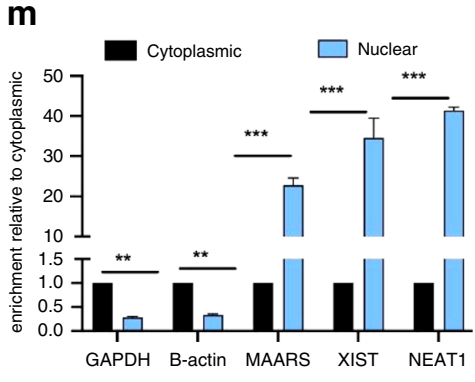

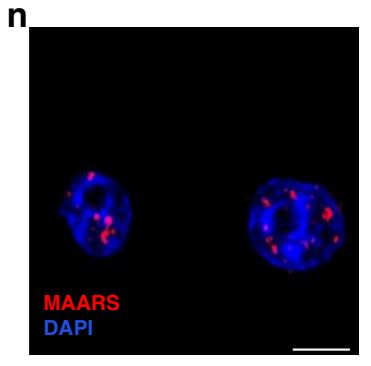

Fig. 1 Identification of the IncRNA MAARS in lesional intima. a RNA derived from aortic intima of LDLR ${ }^{-/-}$mice ( $n=3$; each sample represents RNA pooled from two mice) that were placed on an HCD for 0 weeks (group 1), 2 weeks (group 2), 12 weeks (group 3), and 18 weeks after 6 weeks of resumption of a normal chow diet (group 4). b RNA-Seq results for 11 IncRNA hits obtained by DESeq2/NOR analysis and expressed as fold change compared to group $1(n=3)$. c Heatmap for 11 IncRNAs that were dynamically regulated with progression and regression of atherosclerosis and expression of macrophage markers Mac2, Mac3, and F4/80, as compared to group $1(n=3)$. $\mathbf{d}$ RT-qPCR expression analysis for MAARS in different cell types ( $n=3$ ). e MAARS expression kinetics in macrophages differentiated from bone marrow $(n=3)$. $\mathbf{f}$ MAARS expression in body organs and PBMCs of 24 weeks old C57BL/6 mice $(n=4$, left panel). $\mathbf{g}$ MAARS expression in C57BL6 spleen fractions isolated with magnetic beads specific for different cell types ( $n=3$ ): F4/80 (macrophages), CD8a (T cells), CD11c (dendritic cells), CD19 (B cells), and negative selection for B cells. $\mathbf{h}$ MAARS expression in body organs and PBMCs of 24 weeks old LDLR ${ }^{-/-}$mice fed with high cholesterol diet (HCD) for 12 weeks $(n=5)$. i MAARS expression in 50,000 cells FACS sorted for F4/80- and CD11b-negative or double-positive cells isolated from aorta of ApoE ${ }^{-/}$- mice fed HCD for 12 weeks (progression) and 18 weeks after 6 weeks of resumption to a normal chow diet (regression) $(n=5)$. j RNA-in situ hybridization for MAARS-probe (red), Mac-2 (green), and DAPI (blue) staining in the aortic lesions of $\mathrm{LDLR}^{-/}$- fed HCD for 12 weeks. $\mathbf{k}$ Coding potential assessing tool (CPAT) predicts very low coding potential for MAARS IncRNA. I To test the coding potential, MAARS sequence was cloned upstream of 3xFlag-Tag cassette, transfected in 293 T cells, and immunoblotted for Flag antibody. Positive control was provided with the kit ( $n=3$ experiments). $\mathbf{m}$ RT-qPCR analysis for RNA derived from BMDMs separated into cytoplasmic and nuclear fractions and normalized to the cytoplasmic fraction $(n=3)$. $\mathbf{n}$ RNA-in situ hybridization for negative control- and MAARS-probes on PFA-fixed BMDMs. For all panels, values are mean $\pm \mathrm{SD} ;{ }^{\star} p<0.05 ;{ }^{\star \star} p<0.01 ;{ }^{\star \star \star} p<0.001$.

potential, MAARS sequence was cloned upstream of the p3xFLAG-CMV plasmid, transfected in HEK293 cells, and immunoblotted for FLAG Tag, which showed no detectable peptide or protein (Fig. 11). In addition, MAARS is polyadenylated (Supplementary Fig. 1g) and enriched in the nucleus of the macrophages as observed by cell fractionation (Fig. $1 \mathrm{~m}$ ) and by RNA-ISH in bone marrow-derived macrophages (Fig. 1n and
Supplementary Fig. 1e). MAARS is highly expressed in BMDMs with $9.84 \times 10^{8}$ copies $\mathrm{ng}^{-1}$ of RNA and is expressed 2.4 -fold higher than the regularly used housekeeping gene hypoxanthine phosphoribosyltransferase 1 (HPRT) (Supplementary Fig. 1h). Collectively, these results indicate that $M A A R S$ is a macrophageand nuclear-specific IncRNA markedly induced with atherosclerotic progression. 
MAARS silencing reduces progression of atherosclerosis. For $M A A R S$ silencing we designed different LNA-based gapmeRs that showed 70-90\% knockdown efficiency in BMDMs and selected gapmeR \#1 for further studies (Supplementary Fig. 1i). To explore the role of systemically delivered MAARS-gapmeRs in atherosclerosis, LDLR $^{-1-}$ mice were i.v. injected with vehicle control or MAARS gapmeR twice per week $\left(10 \mathrm{mg} \mathrm{kg}^{-1}\right.$ per injection per mouse) over 12 weeks on HCD (Fig. 2a). After 12 weeks on HCD, gapmeR-mediated silencing of MAARS reduced its expression in the aortic intima by $90 \%$ (Fig. 2 b) and in the spleens by $40 \%$ (Supplementary Fig. 4f), while MAARS could not be detected in the aortic media and the other organs (Fig. 1h and Supplementary Fig. 1b). Furthermore, the MAARS-specific knockdown in the intima was confirmed by RNA-ISH in the aortic sinus lesions, showing an $84 \%$ decrease (Fig. 2c). Remarkably, analysis of atherosclerotic lesion formation by Oil-Red O (ORO) staining revealed a $46 \%$ decrease in lesion area in the aortic sinus and $52 \%$ decrease in the descending thoracoabdominal aorta after knockdown of MAARS (Fig. 2d, e). As shown by immunohistochemistry, MAARS-deficient lesions did not differ in the accumulation of macrophages (Mac-2 and Mac-3), $\mathrm{CD}^{+}{ }^{+}$or $\mathrm{CD}^{+}{ }^{+} \mathrm{T}$ cells, MHCII-positive cells, or vascular smooth muscle cells (Supplementary Fig. 2) after normalization to lesion area.

While MAARS knockdown was associated with a modest reduction in total cholesterol (22\%), LDL (23\%), HDL (20\%), and triglycerides (18\%) (Supplementary Fig. 3a), the lesion areas as quantified by ORO staining were still reduced by $37 \%$ in MAARS knockdown mice when examined in mice with similar total cholesterol in both groups (Supplementary Fig. 3b). Thus, although $\sim 9 \%$ of the atherosclerotic plaque reduction may be accounted for the serum cholesterol level reduction, this cannot entirely explain the substantial reduction in atherosclerosis lesions. Nevertheless, we explored a possible lipid-related mechanism impacting plaque formation and found that MAARS can also affect macrophage cholesterol handling. MAARS knockdown in BMDMs increased cholesterol efflux to the main cholesterol acceptors, HDL, ApoA-1, and to normal serum (Supplementary Fig. $3 c$ ). These data suggest that the modest contribution to the plaque reduction by MAARS deficiency may be due to cholesterol handling. a

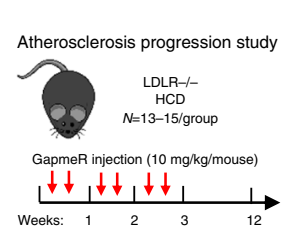

d

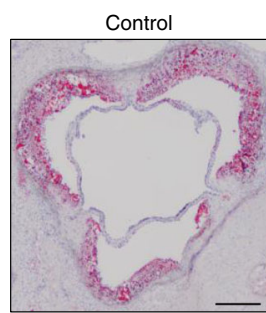

b

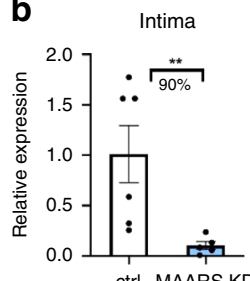

ctrl MAARS KD

MAARS KD

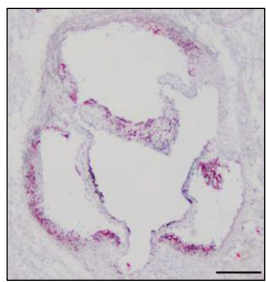

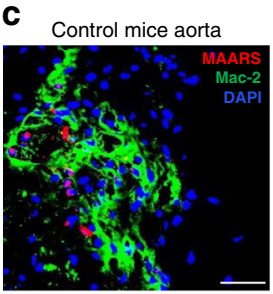

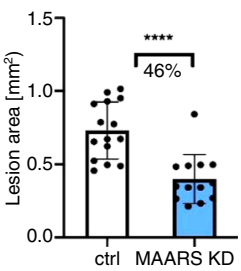

MAARS KD mice aorta

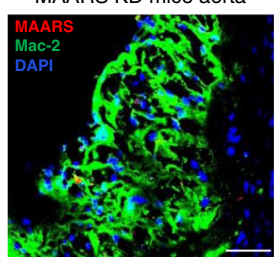

e

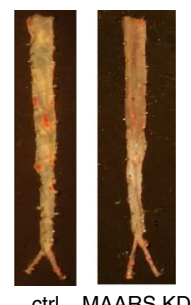

ctrl MAARS KD

MAARS KD

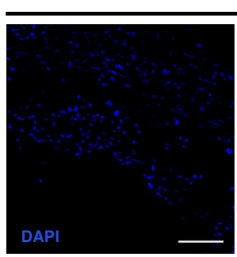

Control
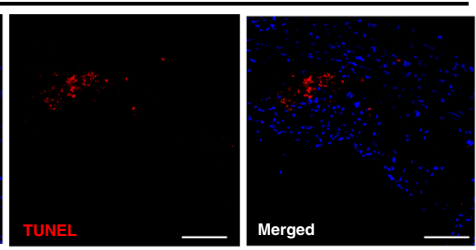

Control
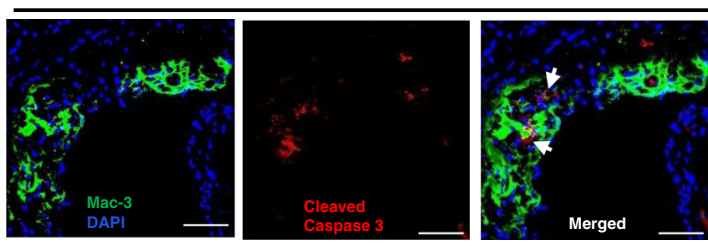
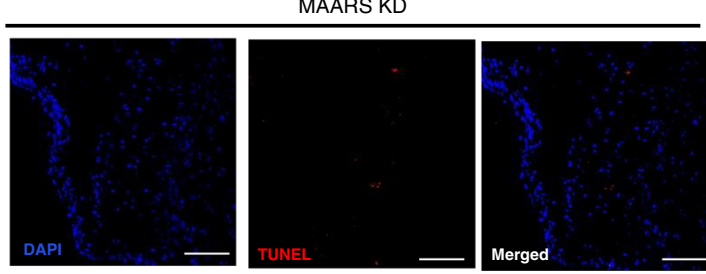

MAARS KD

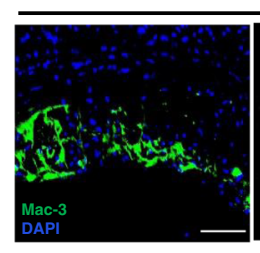

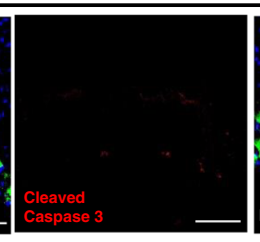
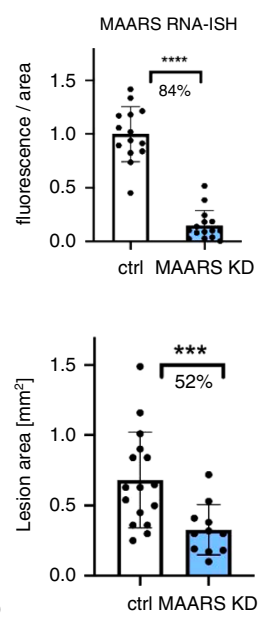

Fig. 2 In vivo knockdown of MAARS inhibits atherosclerotic lesion formation. a $L D L R^{-/}$- mice were i.v. injected with vehicle control gapmeR ( $n=15$ ) or MAARS gapmeR $(n=13)$ twice per week $\left(7.5 \mathrm{mg} \mathrm{kg}^{-1}\right.$ per injection per mouse) and placed on HCD for 12 weeks. b Silencing of MAARS was assessed by RT-qPCR in RNA derived from the aortic intima ( $n=6$ for control gapmeR group and $n=5$ for MAARS gapmeR group). c RNA-ISH representative images and quantification of MAARS knockdown in Mac2 co-stained aortic sinus from control and MAARS KD groups of mice. Representative images and quantification for Oil-Red O staining in the lesions of aortic sinus (d) and in the descending aorta (e) of the control $(n=15)$ and MAARS KD ( $n=13)$ mice. f TUNEL (red) staining was detected in the aortic sinus of the control $(n=15)$ and MAARS knockdown (KD, $n=13)$ groups of LDLR ${ }^{-/-}$mice fed HC, counterstained with DAPI (blue), and quantified as total red fluorescent staining per lesion area. $\mathbf{g}$ The aortic sinus was co-stained with cleaved caspase-3 (red) and Mac-3 antibodies (green) and the Mac3-associated caspase-3 fluorescence was quantified in the aortic sinus of the control ( $n=15)$ and MAARS $\mathrm{KD}(n=13)$ mice. For all panels, values are mean $\pm \mathrm{SD} ;{ }^{{ }^{*}} p<0.05 ;{ }^{\star \star} p<0.01 ;{ }^{\star \star \star} p<0.001 ;{ }^{\star \star \star \star} p<0.0001 ;{ }^{\star \star \star \star \star} p<0.00001$. 
Although MAARS was undetectable in PBMCs, lesional macrophages are in part differentiated from recruited PBMCs, thereby monocyte polarization was analyzed for Ly6C inflammatory expression by FACS in both PBMCs and the spleen. No differences were observed in the anti-inflammatory Ly6C ${ }^{\text {low }}$ or the pro-inflammatory Ly6Cinterm or Ly6 $C^{\text {high }}$ fractions in the spleens and PBMCs of the MAARS-knockdown group compared to vehicle control (Supplementary Fig. 4a). While antisense oligonucleotides can induce liver toxicity ${ }^{18}$, MAARS in vivo knockdown had no significant effect on mouse body weights and the aspartate transaminase (AST) serum levels (Supplementary Fig. $4 b, c)$. Although there were modest increases in the alanine transaminase (ALT) levels, these were in the normal accepted physiological range for $\mathrm{C} 57 \mathrm{BL} / 6$ mice, e.g., below $70 \mathrm{IU} \mathrm{l}^{-1}$ (Supplementary Fig. 4d) ${ }^{19,20}$. RNA expression of MCP-1, IL-6, and TNF- $\alpha$ pro-inflammatory markers were reduced in aortic lesions, but there were no differences observed in the expression of inflammatory genes IL- $1 \beta$ and COX-2 in the aortic intima (Supplementary Fig. 4e). Furthermore, only MCP-1 was reduced in the spleens of MAARS knockdown group, while IL-1 $\beta$, IL-6, TNF- $\alpha$, and MIP-1B were not significantly regulated (Supplementary Fig. 4g). Importantly, there were no regulatory effects on the activity of pro-inflammatory signaling pathways NF-K $\beta$, p38and ERK1/2-MAPK, Akt, or JNK in BMDMs after MAARS gapmeRs transfection (Supplementary Fig. 4h). Taken together, these findings indicate that MAARS deficiency markedly reduced atherosclerotic lesion formation with a mild effect on inflammatory markers and lipid metabolism, and with acceptable liver toxicity.

MAARS knockdown decreases macrophage apoptosis. To investigate additional mechanisms by which neutralization of $M A A R S$ reduced atherosclerotic lesion formation, we investigated the possibility that MAARS could affect lesional apoptosis. Accumulating studies highlight that apoptosis of lesional macrophages plays a crucial role in atherosclerosis, dependent on the stage of progression ${ }^{5,21}$. Investigation of lesion apoptosis by terminal deoxynucleotidyl transferase dUTP nick-end labeling (TUNEL) assay showed a significant reduction in apoptosis (by $52 \%)$ in the aortic sinus of the MAARS-knockdown group (Fig. 2f). Moreover, co-staining of Cleaved Caspase-3 and macrophage marker Mac-2 revealed a decrease of apoptotic macrophages (by 55\%) in the lesions (Fig. 2g). To further assess whether $M A A R S$ directly regulates apoptosis in BMDMs, we performed $M A A R S$ knockdown in the presence and absence of apoptotic stimuli. Indeed, MAARS knockdown dramatically reduced the levels of cleaved Caspase- 8 and -3 in BMDMs treated with highdose TNF- $\alpha$ to trigger apoptosis, and of cleaved Caspase-8, -9, and -3 in BMDM stimulated with Camptothecin (CPT), a topoisomerase I inhibitor that activates apoptosis by inducing DNA damage (Fig. 3a-e) ${ }^{22}$. Similar results were obtained when $M A A R S$ was silenced with a different MAARS gapmeR (Supplementary Fig. 5h). In contrast, MAARS overexpression in BMDMs using a lentivirus system increased the protein levels of cleaved Caspase-3 and -8 (Fig. 3f). In a complementary approach, $M A A R S$ silencing decreased macrophage apoptosis by $50 \%$ as quantified by the TUNEL assay in CPT-treated BMDMs (Fig. $3 \mathrm{~g}$ ). Finally, we used the ApoLive-Glo Caspase-3/7 enzymatic assay that detects the cleavage of Caspases-3 and -7 and observed decreased levels of luciferase activity in the MAARS-knockdown macrophages by $30 \%$ under basal conditions and by $40 \%$ in CPTtreated cells (Fig. 3h). Collectively, these findings suggest that $M A A R S$ has potent effects on regulating macrophage apoptosis in vitro and in vivo.
MAARS interacts with HuR (ELAVL1) to regulate apoptosis. Because lncRNAs typically exert effects in cis or in trans, we first explored the possibility that MAARS knockdown might alter neighboring genes. However, we found no impact of MAARS knockdown for regulation of its neighboring genes (Supplementary Fig. 1j), suggesting a potential trans mechanism. To identify potential MAARS-interacting proteins that may inform mechanisms underlying the decreased progression of atherosclerosis in MAARS-deficient LDLR $^{-1-}$ mice, biotin-labeled T7 in vitro transcribed MAARS or LacZ were incubated with nuclear protein lysates of RAW264.7 macrophages (Fig. 4a). Peptides that specifically bound to biotin-labeled MAARS transcript were identified by liquid chromatography-mass spectrometry (LC-MS/ $\mathrm{MS}$ ) analysis, capturing $\mathrm{HuR}$ ( $\mathrm{Hu}$ antigen $\mathrm{R}$, also known as ELAVL1, Embryonic Lethal, Abnormal Vision, Drosophila-Like 1) as a MAARS-binding protein (Fig. $4 \mathrm{~b}$ and Supplementary Fig. 5a). The HuR protein was only detectable in the eluate of biotin-labeled MAARS as well as in the input BMDM nuclear protein or whole cells lysates (Supplementary Fig. 5b) compared to the LacZ negative control $(n=3)$. Similar HuR pulldown results were observed using 3-end biotinylated MAARS (Supplementary Fig. 5c). As HuR is an RNA-binding protein that interacts with specific ARE we identified $14 \mathrm{HuR}$-specific AREs in the MAARS transcript (Table 1$)^{23-26}$. To investigate whether $\mathrm{HuR}$ is interacting with MAARS by binding to specific ARE motifs, we mutated these AREs and performed RNA pulldown (Fig. 4c, d). Remarkably, the HuR pulldown was dramatically decreased by $81 \%$ when nuclear protein lysates were incubated with ARE-mutated MAARS, as compared to the wild type (WT) $M A A R S$ transcript (Fig. 4d). To further confirm the ARE-specific binding of MAARS to HuR, we performed competition pulldown studies using biotinylated MAARS and ARE synthetic RNA oligonucleotides and observed a concentration-dependent inhibition of MAARS binding to HuR starting with $0.5: 1$ picomole ratio of ARE oligos to biotinylated MAARS (Fig. 4e). Furthermore, the ARE-specific binding of MAARS to HuR was confirmed in vivo after i.v. injection of biotin-labeled AREs-mutated and WT MAARS: HuR was recovered in aortic protein lysates of biotinlabeled WT MAARS injected $\mathrm{ApoE}^{-1-}$ mice fed $\mathrm{HCD}$ for 12 weeks, while it was absent in the ARE-mutated MAARS group (Fig. 4f). Successful delivery of biotin-labeled MAARS was verified by RT-qPCR (Supplementary Fig. 5d). In reverse-pulldown experiments, MAARS expression was increased by fivefold in RNA isolated after HuR immunoprecipitation (IP) compared to IgG control; this was specific to MAARS, compared to other nuclear transcripts such as Histone 3 (Fig. $4 \mathrm{~g}$ ). These data indicate that MAARS interacts with HuR both in vitro and in vivo.

A range of apoptotic stimuli (e.g., staurosporine, doxorubicin, prostaglandins) regulate $\mathrm{HuR}$ shuttling from the nucleus to the cytosol $^{27-30}$. Here, we observed that in macrophages stimulated with CPT, MAARS shuttled together with HuR from the nucleus to the cytosol (Fig. 4h). Additionally, confocal microscopy and fractionation studies highlighted a cytosolic accumulation of $\mathrm{HuR}$ after MAARS knockdown using different gapmeRs (Fig. 4i-l and Supplementary Fig. 6a). Moreover, rescue experiments showed decreased HuR cytosolic shuttling after MAARS WT overexpression using lentivirus while lenti-AREmut had no significant effect (Supplementary Fig. 6b). While HuR silencing did not affect MAARS intracellular localization (Supplementary Fig. 6c), we propose a MAARS tethering mechanism of HuR in the nucleus. This interaction did not involve transcriptional or translational regulation of $\mathrm{HuR}$, since MAARS silencing did not affect HuR expression at the mRNA level (Supplementary Fig. 6d, e) or in whole lysates of BMDMs (Fig. 4j). However, after MAARS knockdown we observed increased HuR expression in the 
a
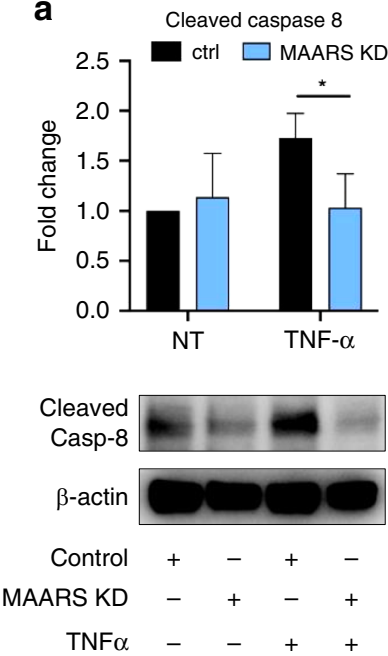

e
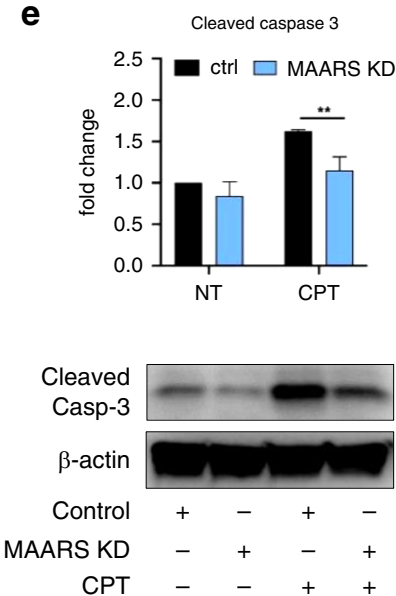

b
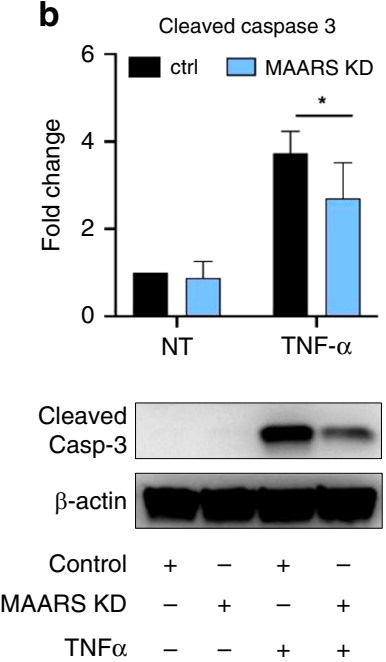

C
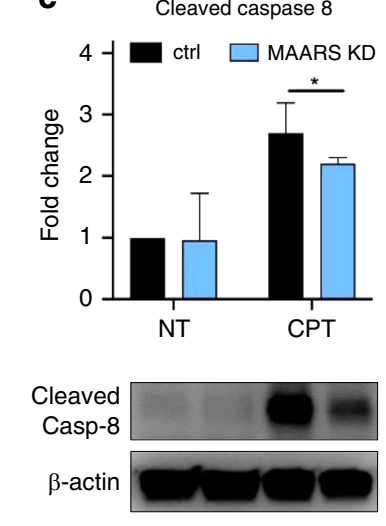

Control + - + -

MAARS KD - + - +

CPT - -++
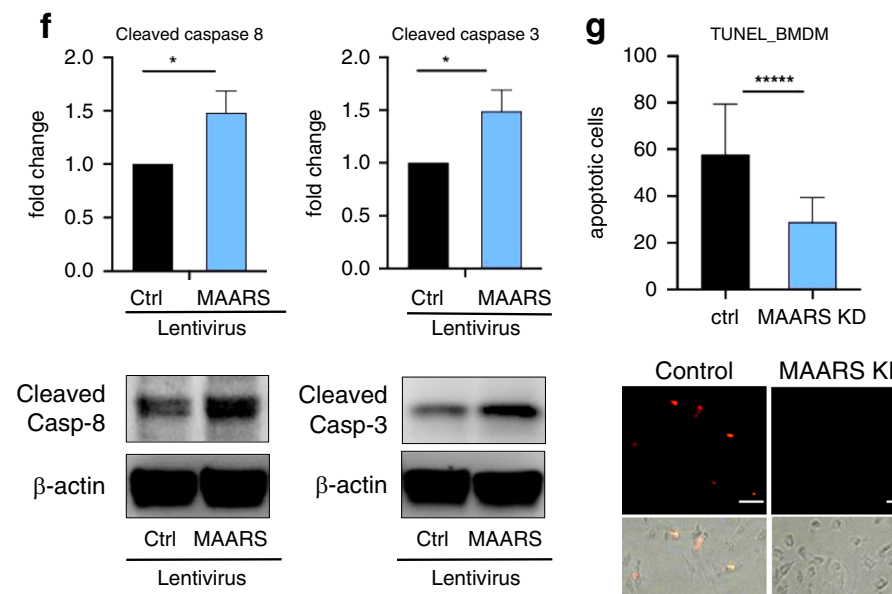

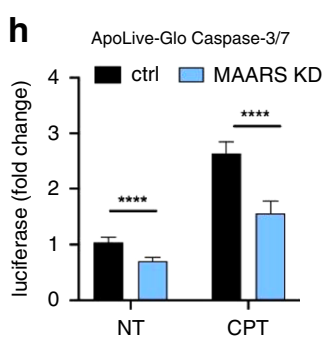

d
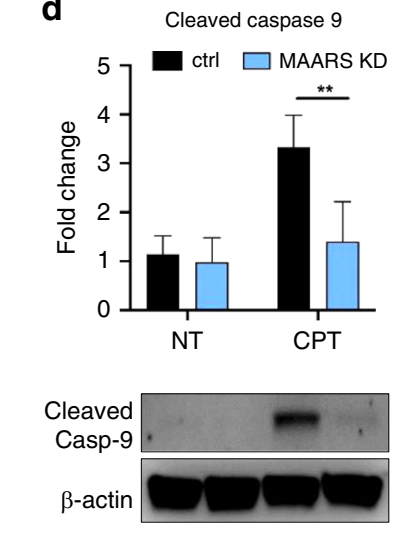

Control + - + -

MAARS KD $-++\quad+$

СPT - -++

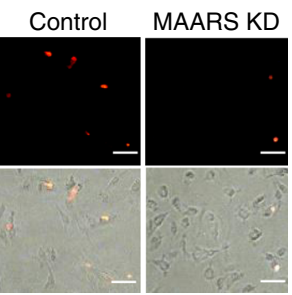

Fig. 3 MAARS knockdown decreases macrophage apoptosis. Western blot quantification of cleaved caspase-8 (a) and cleaved caspase-3 (b) lysate from BMDMs transfected with MAARS-specific or control gapmeRs, and activated with TNF-alpha $\left(50 \mathrm{ng} \mathrm{ml}^{-1}\right)$ for $18 \mathrm{~h}(n=4)$. Western blot quantification of cleaved caspase-8 (c), cleaved caspase-9 (d), and cleaved caspase-3 (e) from lysates of BMDMs transfected with MAARS-specific or control gapmeRs, and activated for $2 \mathrm{~h}$ with $20 \mu \mathrm{M}$ camptothecin (CPT; $n=3)$. f Cleaved caspase- 3 and -8 expression in BMDMs transduced with lentivirus control or overexpressing MAARS $(n=3)$. $\mathbf{g}$ In vitro TUNEL staining of BMDMs transfected with MAARS-specific and control gapmeRs and activated with $20 \mu \mathrm{M}$ CPT $(n=3 ; 25$ images quantified per condition). $\mathbf{h}$ Quantification of apoLiveGlo cleaved caspase-3/7 enzymatic assay of BMDMs transfected with MAARSspecific and control gapmeRs and activated with or without CPT $(20 \mu \mathrm{M})(n=3)$ For all panels, values are mean $\pm \mathrm{SD}^{*}{ }^{\star} p<0.05$; ${ }^{\star \star} p<0.01$; ${ }^{\star \star \star} p<0.001$; ${ }^{\star \star \star \star} p<0.0001 ;{ }^{\star \star \star \star \star \star} p<0.00001$.

cytosolic fraction and decreased expression in the nuclear fraction of BMDMs treated with or without CPT, suggesting that MAARS silencing regulates the $\mathrm{HuR}$ cytosolic shuttling (Fig. 4k, 1). Moreover, HuR shuttling in the cytosol in response to MAARS gapmeR was concentration-dependent,(Supplementary Fig. 6f). HuR is an important RNA-binding protein that regulates apoptosis by binding to the mRNA of pro- and anti-apoptotic genes and mediating their stability or trafficking to the cytosol, among other mechanisms ${ }^{12,16}$. Importantly, MAARS knockdown regulated several well-described mRNA targets of $\mathrm{HuR}$, decreasing the expression of p53, p27, and Caspase- 8 and -9 in BMDMs (Fig. 5a) and of p27, Caspase-8, -9, and BAX in the intima of LDLR $^{-1-}$ mice (Fig. 5c). In contrast, MAARS silencing increased the expression of anti-apoptotic genes BCL2, Prothymosin A (ProtA), Mcl-1, and SIRT1 in BMDMs (Fig. 5b) and of BCL2, ProtA, and SIRT1 in the intima of $\mathrm{LDLR}^{-1-}$ mice (Fig. 5c). Similar trends were observed in the spleens of $\mathrm{LDLR}^{-1-}$ mice after MAARS knockdown (Supplementary Fig. 6h), while no effects were observed in the aortic media fraction (Supplementary
Fig. 6g). Consistently, MAARS silencing strongly regulated the mRNA stability of HuR targets, decreasing p53, caspase-8, -9, and p27, and increasing BCL2 after activation with CPT and treatment with the transcriptional inhibitor Actinomycin D (Fig. 5d). The mRNA stability of Histone 3, which is not a known HuR target, was not affected by MAARS knockdown, demonstrating specificity for HuR targets (Fig. 5d). RNA IP experiments in lysates from BMDM treated with MAARS or control gapmeRs confirmed the HuR-dependent effect on selected apoptosis genes: MAARS knockdown increased the HuR binding to Mcl1, SIRT1, ProtA, and Bcl-2 mRNAs, suggesting a potential MAARS buffering effect on HuR (Fig. 5e). Moreover, in MAARS knockdown BMDMs, lentiviral overexpression of WT MAARS rescued HuR binding to its targets by RIP in comparison to MAARS AREmut lentiviral overexpression (Supplementary Fig. 6i). In addition, dependency studies of silencing both HuR and MAARS showed that the increase in cleaved caspase-3 observed after HuR knockdown was abrogated in the presence of MAARS deficiency (Fig. 5f). Interestingly, although CPT and 
a

b
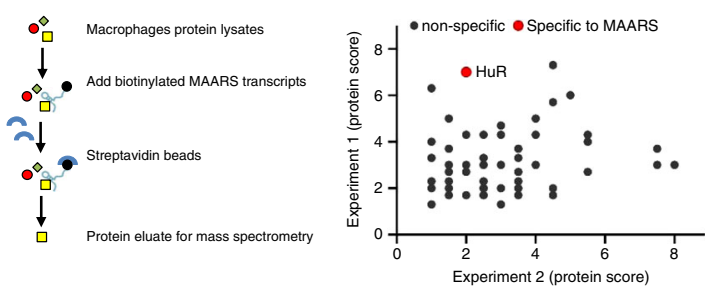

e
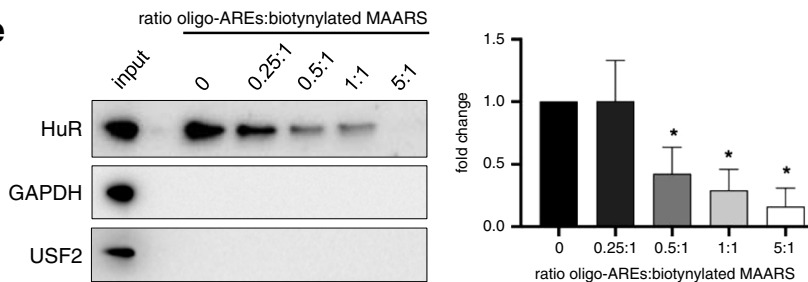

C

d ratio oligo-AREs:biotynylated MAARS MAARS HUR ARE motifs
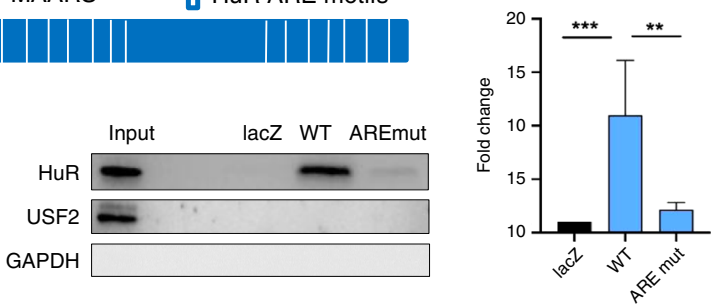

f
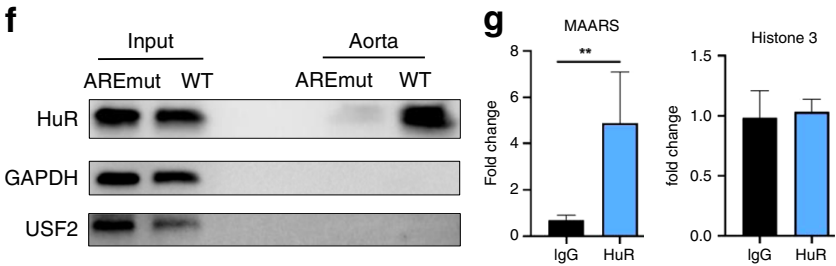

h
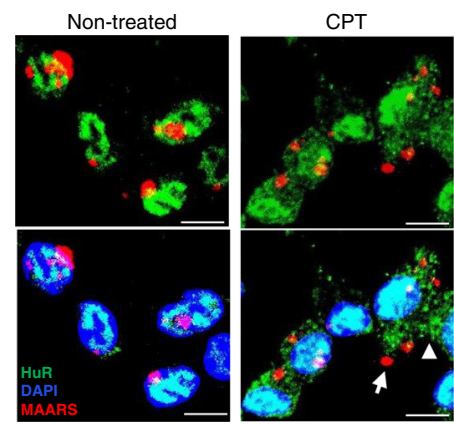

j

Whole cell lysates
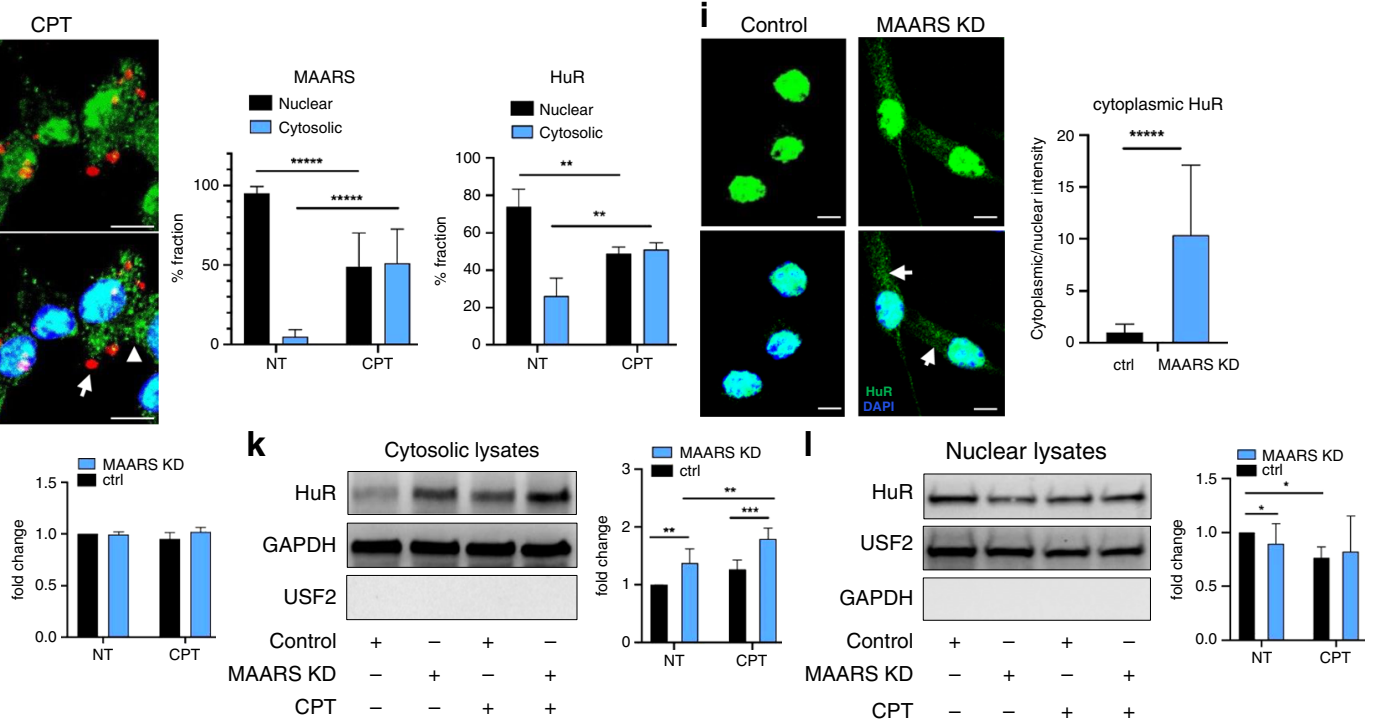

Fig. 4 MAARS interacts with HuR (ELAVL1). a Illustration of IncRNA pulldown using nuclear protein extract from RAW264.7 macrophages for in vitro transcribed biotin-labeled MAARS or negative control LacZ followed by streptavidin beads pulldown. Protein eluate was sent for MS. $\mathbf{b}$ Proteins identified in pulldowns of biotynlated MAARS from two independent experiments, two technical replicates. $\mathbf{c}$ Schematic representation of HuR binding motifs to AU-rich elements (ARE) on MAARS transcript. d Biotinylated IncRNA pulldown using MAARS wild type (WT) or MAARS with mutated HuR binding ARE sequences (AREmut) in nuclear lysates of BMDMs $(n=5)$. e Competition pulldown using biotinylated MAARS and RNA synthetic AREs oligonucleotides at different picomole ratios $(n=3)$. $\mathbf{f}$ In vivo IncRNA pulldown using whole cell lysates from aorta of ApoE ${ }^{-/-}$mice fed HCD following two i.v. injections of biotinlabeled IncRNA transcripts ( $n=2$ per group, pooled). g RNA immunoprecipitation (RIP) in nuclear BMDM lysates using HuR-specific antibody ( $n=3$ ). h BMDM treatment with camptothecin (CPT) induces HuR (arrow head) and MAARS (arrow) shuttling from the nucleus to the cytosol. Representative images and quantification of cytosolic and nuclear HuR and MAARS staining in BMDM treated with or without camptothecin (CPT, $20 \mu M)$ for $1 \mathrm{~h}(n=3$, 200 cells quantified per condition). i HuR cytosolic (white arrows) and nuclear staining was quantified in confocal microscopy images of BMDMs transfected with control or MAARS gapmeRs ( $n=3,100$ cells quantified per condition). $\mathbf{j}$ Quantification of Western blots of HuR in whole cell lysates from BMDMs transfected with control or MAARS gapmeRs treated with or without CPT (20 $\mu \mathrm{M}, 1 \mathrm{~h})$. Expression of HuR in cytosolic (k) and nuclear fractions (I) from BMDMs transfected with control or MAARS gapmeRs treated with or without CPT $(20 \mu \mathrm{M}, 1 \mathrm{~h})(n=6$, values are mean \pm SD). For all panels values are mean $\pm \mathrm{SD}$. ${ }^{\star} p<0.05 ;{ }^{\star \star} p<0.01 ;{ }^{\star \star \star} p<0.001 ;{ }^{\star \star \star \star *} p<0.00001$.

TNF- $\alpha$ decrease MAARS expression, potentially due to a feedback mechanism (Supplementary Fig. 5e, f), the MAARS binding affinity to HuR is not affected by CPT and TNF- $\alpha$ treatment in BMDMs as observed in the pulldown studies (Supplementary Fig. 5g). Taken together, these findings indicate that lncRNA MAARS actively binds the RNA-binding protein HuR, which in turn regulates the expression of a range of apoptosis genes in macrophages. Loss of MAARS in lesions thereby facilitates a protective anti-apoptotic program associated with regulation of
mRNA stability of HuR targets and downstream decrease of caspase- $8,-9$, and -3 cleavage.

MAARS knockdown regulates plaque necrosis and efferocytosis. Plaque necrosis has been linked to alterations in the presence of lesional apoptotic cells $s^{3,6}$. We observed a lower number of necrotic cores in $\mathrm{LDLR}^{-/-}$mice treated with MAARS-specific gapmeRs (by 60\%) compared to $\mathrm{LDLR}^{-1-}$ mice treated with gapmeR controls (Fig. 6a). Necrotic core formation is associated 
Table 1 AU-rich elements identified in the MAARS transcript and the respective mutations in the AREmut biotinylated MAARS transcript used for RNA pulldown in Fig. 4d-f.

\section{AU-rich elements}

TTTTTGT

AATTTTA

TTTTA

TTTTTAA

TTTATTT

TTTTTGTTT

TTTGTTT

TTTAATT

AATATTT

ATTTTC

CTTTTAT

ATTTA

TATTTATT

TTTCCTT

with defective efferocytosis, or the ability of macrophages to engulf and degrade apoptotic cells before they become necrotic ${ }^{3,31}$. Macrophage MerTK (c-Mer tyrosinekinase) serves as a cell surface receptor and signaling molecule mediating efferocytosis, and MerTK has a central role in promoting efferocytosis and decreasing necrotic core formation in atherosclerotic lesions ${ }^{32}$. MAARS silencing in BMDMs incubated with Jurkat apoptotic cells increased MerTK surface expression as observed by flow cytometry (Fig. 6b and Supplementary Fig. 7a) and confocal microscopy (Fig. 6c). Furthermore, lesional macrophages (co-stained with Mac3) of $\mathrm{LDLR}^{-1-}$ mice treated with MAARS gapmeRs had increased expression of MerTK (by 49\%) compared to control gapmeR injected mice (Fig. 6d). In a different approach to evaluate in vivo macrophage efferocytosis, aortic roots were co-stained with TUNEL and macrophage marker Mac-3 and the ratio of free vs. macrophage-associated apoptotic cells was assessed as previously described $^{33}$. This ratio was markedly decreased by $53 \%$ in the atherosclerotic lesions of MAARS-knockdown mice, indicating more efficient efferocytosis (Fig. 6e). To further assess the role MAARS in macrophage efferocytosis in vitro, we used lentiviral overexpression studies. BMDMs overexpressing MAARS decreased efferocytosis of calcein-stained apoptotic Jurkat cells (Supplementary Fig. 7b). Conversely, in CPT-treated BMDMs, MAARS knockdown using two different gapmeRs significantly increased efferocytosis (by 72\%) while HuR silencing had the opposite effect (39\% decrease). In addition, silencing both MAARS and HuR showed that the MAARS-mediated effect on efferocytosis is dependent upon HuR. (Fig. 6f and Supplementary Fig. 7c). Further assessments by FACS or Western Blot of other mediators of efferocytosis such as dynamin-related protein 1, CD47 or CD172 (Serpin- $\alpha$ ) did not show any significant regulation after MAARS silencing in BMDMs (Supplementary Fig. 7d, e).

To explore the pathways and biological processes affected by $M A A R S$ knockdown, unbiased genome-wide RNA-Seq was performed on MAARS gapmeR-transfected BMDMs. A number of dynamically regulated genes were identified $(p<0.0001)$ (Supplementary Fig. 8a). Network pathway analysis using MetaCore showed that the top up- and downregulated pathways were mostly related to apoptosis, inflammation, and efferocytosis: four apoptosis-related pathways were downregulated while pathways related to phagocytosis, a debris-cleaning process similar to efferocytosis, and cytoskeleton rearrangement pathways were upregulated (Supplementary Fig. 8a). Moreover, process network analysis of downregulated pathways indicated many apoptosis- related genes such as caspases, Bak, Bax, Bid, Bcl-XL, or cytochrome C (Supplementary Fig. 8b), while part of the upregulated genes in the network play roles in efferocytosis and cytoskeleton rearrangement, e.g., Profilin, Alpha-actin, Tubulin, ROCK, and Rho GTPases (Supplementary Fig. 8c). Taken together, these findings indicate that MAARS knockdown decreases macrophage apoptosis and increases their clearance, or efferocytotic capacity, thereby decreasing plaque necrosis in the atherosclerotic lesions.

\section{Discussion}

Macrophage apoptosis occurs during all stages of atherosclerosis ${ }^{34,35}$. While some studies using different mouse models indicate that macrophage apoptosis in the early phases of atherosclerosis may be protective $\mathrm{e}^{36-40}$, the accumulation of apoptotic debris in later stages, together with defective macrophage efferocytosis, may lead to the so-called "postapoptotic" or "secondary" necrosis and ultimately to atherosclerotic plaque progression $^{21}$. Herein, we provide evidence that the macrophagespecific lncRNA MAARS regulates apoptosis by interacting with $\mathrm{HuR}$, an RNA-binding protein that is a critical mediator of transcript stability and apoptosis. In support, lncRNA MAARS expression was potently induced in intimal lesions during the progression phase after 12 weeks of $\mathrm{HCD}$ in $\mathrm{LDLR}^{-/-}$mice, a time point associated with a marked increase in lesional macrophage apoptosis and plaque necrosis along with reduced efferocytosis (Figs. 2f, g and 6a, d, e). Furthermore, neutralization of $M A A R S$ in the intima of lesions profoundly reduced plaque areas in the aortic sinus and thoracoabdominal aortas of $\mathrm{LDLR}^{-/-}$ mice (Fig. 2d, e). Importantly, these effects were predominantly independent of differences between groups in the lipoprotein profile (Supplementary Fig. 3b), lesional accumulation of leukocyte subsets (Supplementary Fig. 2), or inflammatory signaling pathways (Supplementary Fig. 4h), indicating that MAARS deficiency regulated a distinct pathway. Further interrogation of $M A A R S$-deficient lesions revealed marked reduction in macrophage apoptosis and increased lesional efferocytosis. Mechanistically, MAARS deficiency reduced apoptotic markers such as p53, p27, and the caspase- $3,-8$, and -9 while increasing antiapoptotic markers BCL2, Mcl1, and ProtA by interacting with HuR. In light of these findings, these studies identify MAARS as a potent regulator of $\mathrm{HuR}$ and macrophage apoptosis. In dependency studies, we show that HuR silencing induced apoptosis in macrophages, while MAARS knockdown can abrogate this phenotype as observed in the cleavage of caspase-3 (Fig. 5e).

This study highlights how cell-specific silencing of a lncRNA in the intima of atherosclerotic plaques using the nuclease-resistant LNA gapmeRs can exert powerful phenotypic switching. Other gapmeRs to lncRNAs have been recently described to regulate cardiac hypertrophy ${ }^{41,42}$. These studies demonstrated that delivery of gapmeRs can have cell-specific effects depending on the route of delivery and relative expression of interactors in target cells. In keeping with this, MAARS deficiency reduced macrophage apoptosis in the intima, but not in the media of aortic lesions (Fig. $2 \mathrm{f}$ and Supplementary Fig. 6e). As a consequence of macrophage apoptosis, cells may exhibit impaired homeostatic control of functions important to clearance of cellular debris or apoptotic cells ${ }^{43}$. Consistent with this notion, loss of MAARS in macrophages significantly reduced apoptotic markers and enhanced macrophage-mediated efferocytosis. These findings suggest that $M A A R S$-deficient lesional macrophages are more functional, independent of lipid lowering or direct regulation of leukocyte recruitment to the vessel wall.

LncRNAs can exert their function by interacting with proteins, RNAs, and/or genomic DNA. Nuclear lncRNAs have been widely 

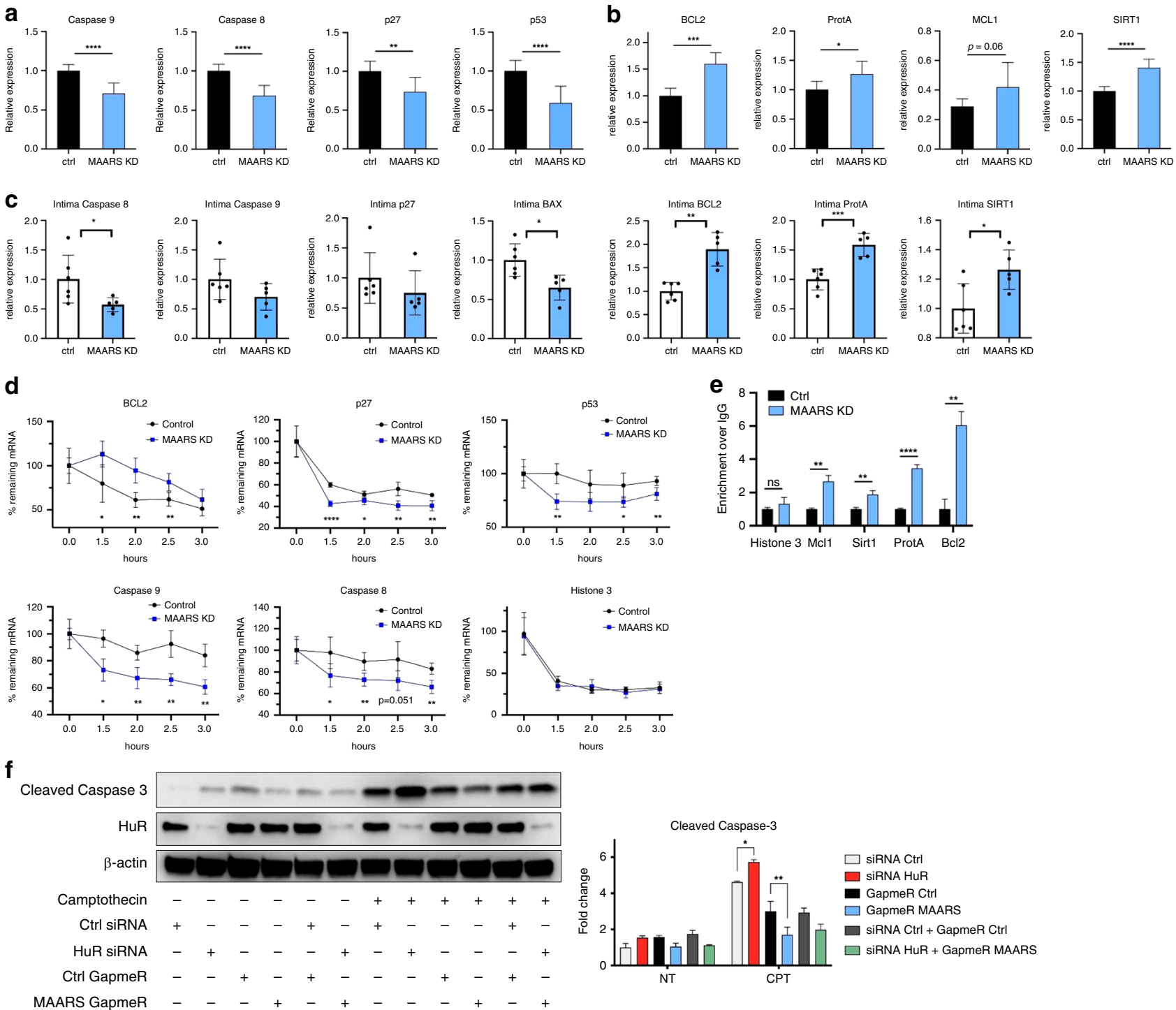

Fig. 5 MAARS knockdown regulates HuR apoptotic targets. a MAARS knockdown decrease pro-apoptosis specific HuR targets in BMDMs transfected with MAARS gapmeRs $(n=4)$. b MAARS knockdown increases anti-apoptosis-specific HuR targets in BMDMs transfected with MAARS gapmeRs $(n=4)$. c Relative expression of HuR targets in the intima of $\mathrm{LDLR}^{-/}$mice treated with control $(n=6)$ and MAARS gapmeR ( $\left.n=5\right)$ (from study in Fig. 2). d mRNA stability studies of HuR targets in BMDMs transfected with control or MAARS gapmeRs and treated with camptothecin (CPT) for $1 \mathrm{~h}$, then activated actinomycin $D\left(100 \mathrm{ng} \mathrm{ml}^{-1}\right)$ for the indicated time points (representative experiment of three independent experiments). e RNA immunoprecipitation (RIP) using HuR-specific antibody in lysates from BMDMs treated with MAARS or control gapmeR (shown is one RIP representative of three independent experiments). $\mathbf{f}$ Dependency studies of MAARS and HuR using the indicated gapmeRs and siRNAs, respectively, on cleaved caspase-3 expression in BMDMs treated with or without $20 \mu \mathrm{M}$ CPT for 2 h. For all panels values are mean \pm SD. ${ }^{\star} p<0.05 ;{ }^{\star \star} p<0.01 ;{ }^{\star \star \star} p<0.001 ;{ }^{* \star \star \star} p<0.0001$.

described to interact with heterogeneous nuclear ribonucleoproteins ${ }^{44}$. In this study, using RNA pulldown studies in combination with LC-MS/MS we have detected HuR/ELAVL1 as an interactor of MAARS. Our in vitro and in vivo data reveal a binding preference of MAARS to HuR, an RNA-binding protein and key regulator of cellular apoptosis ${ }^{15,16}$. The specific interaction of MAARS with HuR was confirmed using two pulldown methods in the BMDM nuclear fraction and in vivo after intravenous injection of biotinylated MAARS in $\mathrm{ApoE}^{-1-}$ mice fed $\mathrm{HCD}^{9,10,45-47}$. HuR is an RNA-binding protein that has been shown to control apoptosis by regulating mRNA stability, modulating translation, or by regulating mRNA nucleocytoplasmic shuttling ${ }^{12,27}$. Previous studies have documented a role of HuR in the stabilization of both pro-apoptotic genes such as p53, p27, caspase- 8 , caspase- 9 , cyclins $\mathrm{A}, \mathrm{B} 1$, and $\mathrm{D} 1^{16,48-53}$, and antiapoptotic genes such as Bcl-2, Mcl-1, and Prothymosin $\alpha^{12,54,55}$, dependent on the stimuli, cell type, and stress intensity. In basal conditions, HuR is stabilizing the mRNA of anti-apoptotic genes such as Bcl-2, Mcl-1, and Prothymosine $\alpha,{ }^{12}$ while HuR knockdown or knockout induces apoptosis ${ }^{56,57}$. However, under lethal stress HuR can have a pro-apoptotic phenotype after its cleavage, dependent on stimuli intensity ${ }^{58}$. Moreover, ATPase helicases regulate HuR ubiquitination and can induce an HuR-dependent destabilization of its mRNA targets such as $\mathrm{p} 21^{59}$. While MAARS expression is increased with progression of atherosclerosis and decreased with regression in aortic macrophages (Fig. 1i), MAARS expression also decreased in BMDMs treated with TNF$\alpha$ and CPT (Supplementary Fig. 5e, f). Several mechanisms can be responsible for the observed regulation in vitro, including compensatory or feedback mechanisms. Indeed, lncRNAs can serve as negative feedback regulators. One example is the lncRNA Mirt2 that is limiting the production of pro-inflammatory cytokines and 
a

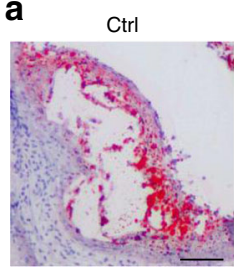

c
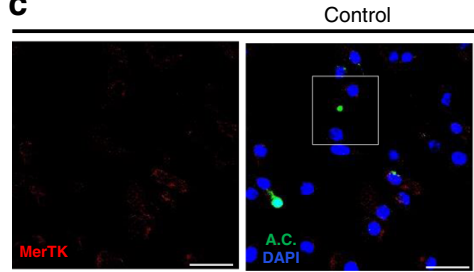

d
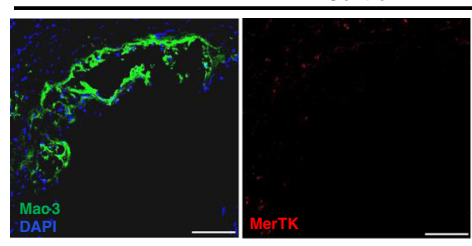

e

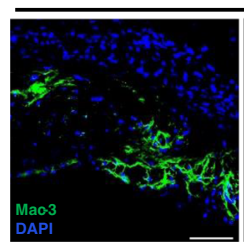

f
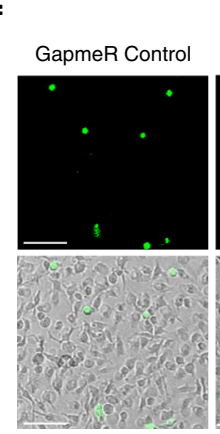
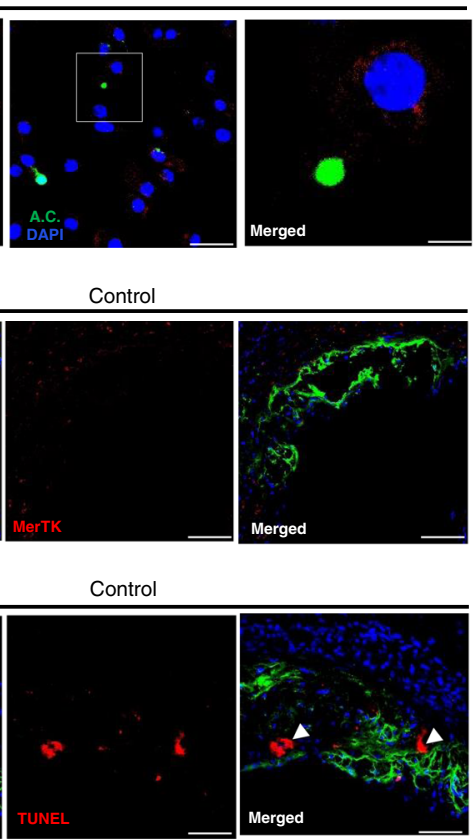

Merged

MAARS KD

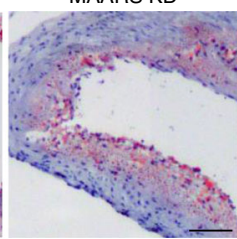

Control
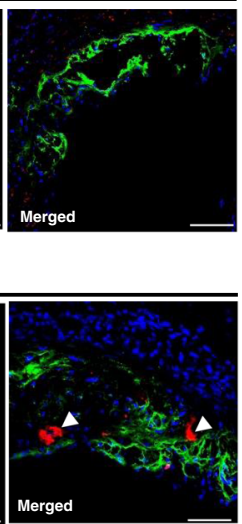

b BMDM non-treated

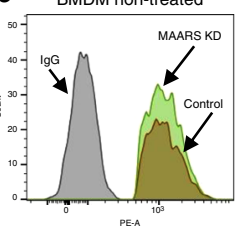

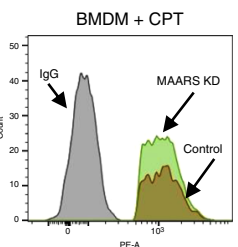

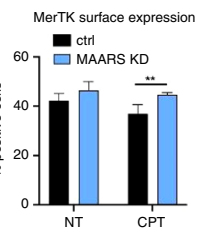

MAARS KD
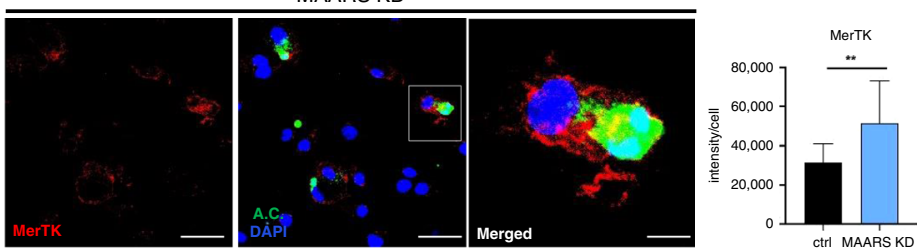

MAARS KD
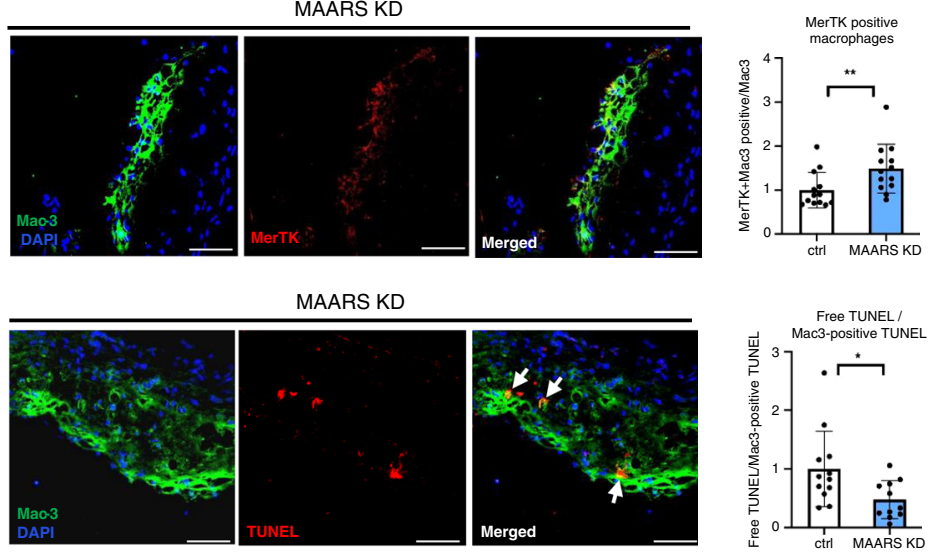

Fig. 6 In vivo knockdown of MAARS inhibits plaque necrosis and increases efferocytosis. a Quantification of plaque necrosis in the lesions of the aortic sinus of LDLR $/-$ mice fed HCD treated with control $(n=15)$ or MAARS gapmers (knockdown $(K D), n=13)$. b Flow cytometry staining with the efferocytosis marker MerTK in BMDMs treated with or without $20 \mu \mathrm{M} \mathrm{CPT}$ for $2 \mathrm{~h}$ and incubated with Jurkat apoptotic cells for $1 \mathrm{~h}$ at a BMDM:AC ratio of 1:10. $\mathbf{c}$ High resolution confocal images and quantification of MerTK staining in BMDMs treated with $20 \mu \mathrm{M} C P T$ for $2 \mathrm{~h}$ and incubated with Jurkat apoptotic cells $(A C)$ for $1 \mathrm{~h}$ at a BMDM:AC ratio of 1:1. d Evaluation of macrophage efferocytosis in vivo by co-staining with MerTK and Mac-3, and quantification of the ratio between double-positive MerTK $+/ \mathrm{Mac}^{+}$and Mac3 staining alone in the aortic sinus of $\mathrm{LDLR}^{-/}-$mice fed HCD treated with control $(n=15)$ or MAARS gapmers (KD, $n=13$ ). e Quantification of the ratio between free TUNEL staining (arrow heads) vs. Mac3-positive TUNEL staining (arrows), reflecting Mac3-positive efferocytotic macrophages, in the aortic sinus lesions LDLR ${ }^{-/-}$mice fed HCD treated with control ( $\left.n=15\right)$ or MAARS gapmeRs $(K D, n=13)$. $\mathbf{f}$ In vitro efferocytosis assay: BMDMs were transfected with control or MAARS gapmeRs in the presence or absence of the indicated control or HuR siRNAs and subsequently incubated with calcein AM-labeled apoptotic Jurkat cells for $1 \mathrm{~h}$. Efferocytosis was quantified as the ratio of calceinpositive $(<3 \mu \mathrm{m})$ vs. calcein-negative macrophages $\left(n=3\right.$, quantification of at least 20 images per condition). For all panels, values are mean \pm SD; ${ }^{\star} p<$ $0.05 ;{ }^{\star \star} p<0.01 ;{ }^{\star \star \star} p<0.001 ;{ }^{\star \star \star \star} p<0.0001 ;{ }^{\star \star \star \star} p<0.00001$.

protects mice from endotoxemia-induced fatality, but is itself increased in response to inflammatory stimuli ${ }^{60}$. While we have not identified a negative feedback mechanism for MAARS, we cannot rule out the existence of such mechanism in response to stress induced by apoptotic stimuli. Importantly, in all our experiments the CPT and TNF- $\alpha$ were added to the cells when the MAARS silencing was already achieved ( 90\%) in BMDMs (Supplementary Fig. 5e, f), hence the potential MAARS downregulation after the addition of CPT and TNF- $\alpha$ was likely negligible. Moreover, the MAARS binding affinity to $\mathrm{HuR}$ is not affected by CPT or TNF- $\alpha$ treatments in BMDMs, as observed in the pulldown experiments (Supplementary Fig. 5g). 


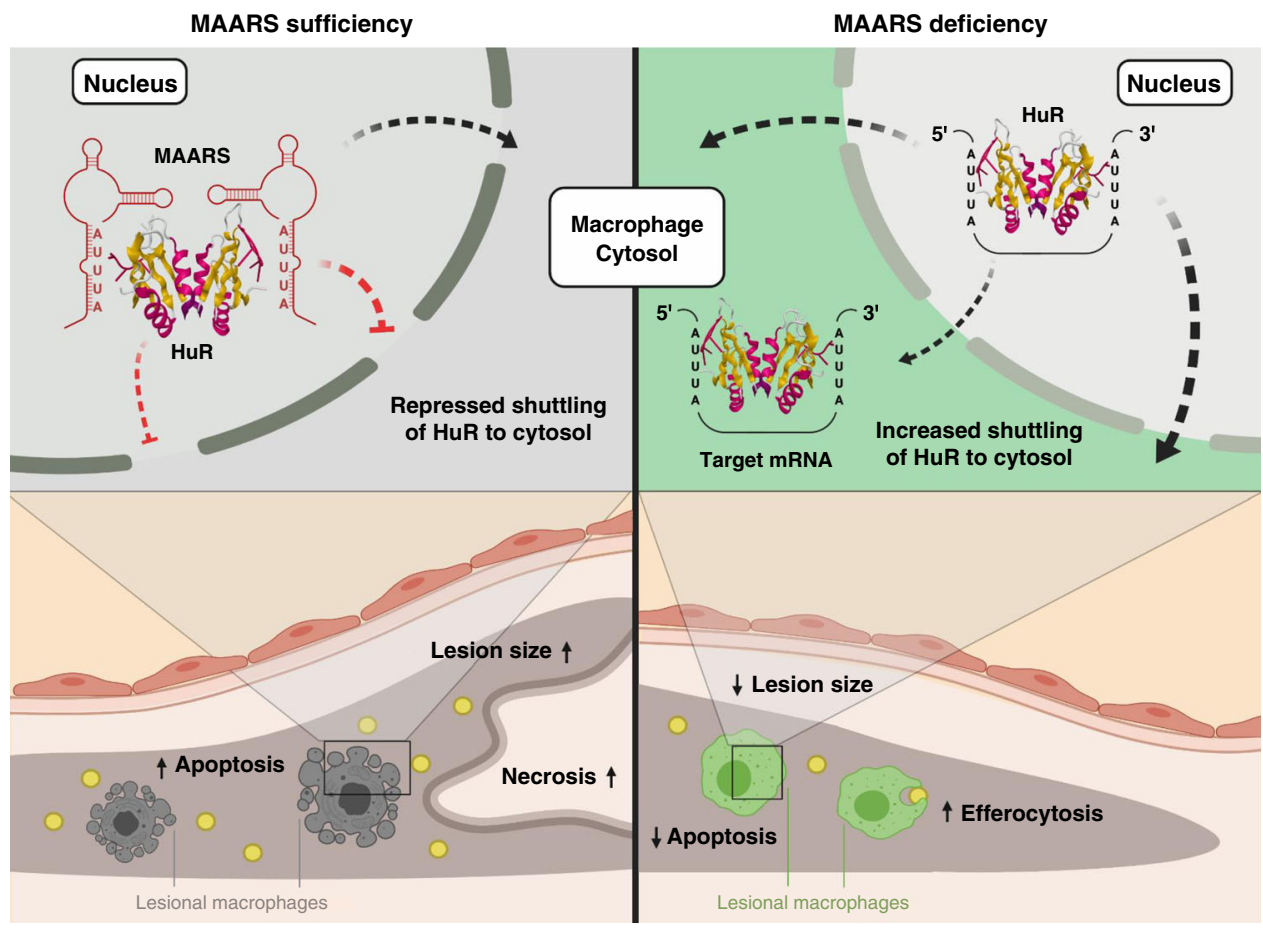

Fig. 7 Proposed mechanism for MAARS regulation of HuR and effects on macrophages apoptosis, efferocytosis, and atherosclerosis. MAARS interacts with HuR (Protein Data Bank structure 6GC5) by tethering it in the macrophage nucleus, preventing its shuttling to the cytosol and interfering with its RNA-stabilizing function. MAARS deficiency induces HuR shuttling in the cytosol, decreasing macrophage apoptosis and increasing macrophage efferocytosis. Consequently, plaque necrosis and atherosclerosis progression is reduced. Created with BioRender.com.

Mechanistically, MAARS knockdown increased the expression of several well-described anti-apoptotic HuR mRNA targets (Bcl2, SIRT1, Prothymosine $\alpha$ ) in vitro and in vivo, while decreasing other pro-apoptotic HuR targets such as p53, p27 and the caspases- 8 and -9 , by regulating their mRNA stability (Fig. $5 a-d$ ). Furthermore, MAARS knockdown increased binding of $\mathrm{HuR}$ to stabilize the anti-apoptotic mRNAs Bcl-2, Mcl1, SIRT1, and Prothymosine $\alpha$, suggesting that MAARS is buffering HuR in the nucleus (Fig. 5e). We show here that HuR interacts with MAARS by binding to specific ARE and mutating these sites in the MAARS transcript can dramatically decrease the MAARS-HuR interaction in vitro and in vivo (Fig. 4e, f). While MAARS tracks with $\mathrm{HuR}$ shuttling in the cytosol after apoptosis stimulation with CPT, MAARS knockdown increased the accumulation of HuR in the cytosol, suggesting that under basal conditions MAARS tethers $\mathrm{HuR}$ in the nucleus, preventing its trafficking to the cytosol, thereby preventing its functional role in mRNA stability (Fig. 4i, j).

Accumulating studies highlight that a reduction in macrophage apoptosis increases their efferocytosis potential and overall clearance of plaques debris, further reducing plaque necrosis $31,61,62$. We observed in this study that MAARS knockdown is associated with increased cell surface expression of MerTK, a key efferocytosis receptor, in vivo in the aortic intima and in BMDMs (Fig. 5a-d). Moreover, MAARS silencing increased the mRNA expression of SIRT1, an anti-apoptotic HuR target gene ${ }^{49}$ that is also known to increase macrophage efferocytosis ${ }^{63,64}$. However, besides its role in apoptosis and efferocytosis, SIRT1 is a key player in other cellular processes such as macrophage self-renewal ${ }^{65}$ or polarization $^{66,67}$. Although we show here that MAARS knockdown strongly induced macrophage efferocytosis by increasing the expression of MerTK and of the HuR gene target SIRT1, there are likely alternative pathways involved in this process. In this study we show that MAARS deficiency in macrophages decreases apoptosis and increases their efferocytosis; consequently, this leads to lower plaque necrosis (Fig. 7). Taken together, these findings indicate that MAARS IncRNA can actively bind to the RNAbinding protein $\mathrm{HuR}$ and mediate apoptosis. These findings provide considerable insights and potential impact for controlling HuR and its nuclear-to-cytoplasmic shuttling that may be dysregulated in diverse disease states.

As MAARS knockdown mice still demonstrated a marked reduction in atherosclerotic lesion formation even after normalization of total cholesterol between groups (Supplementary Fig. 3b), we explored the possibility of a direct effect on cholesterol handling. In support, MAARS knockdown also influenced macrophage cholesterol metabolism, as it increased the cell cholesterol efflux to the main serum acceptors in BMDMs, (Supplementary Fig. 3c). Cholesterol efflux is involved in the regulation of total cell and membrane cholesterol content, both impacting on macrophage functions and atherosclerosis ${ }^{68,69}$. Thus, the impact of MAARS on macrophage cholesterol handling might be another one of the mechanisms of its proatherogenicity. In addition, as previous studies demonstrated that high cholesterol content can induce macrophage apoptosis ${ }^{70-72}$, future studies will be of interest to explore the relationship between the effect of MAARS on macrophage cholesterol metabolism and apoptosis.

In summary, we have identified lncRNA MAARS from intimal atherosclerotic lesions as a homeostatic regulator of macrophage apoptosis and efferocytosis by interaction with HuR. Deficiency of the IncRNA MAARS reduced lesional macrophage apoptosis leading to enhanced efferocytosis, and decreased atherosclerosis largely independent of lipid lowering, lesional leukocyte accumulation, or inflammatory signaling pathways. These findings demonstrate a role of this lncRNA as an interactor of an RNAbinding protein, $\mathrm{HuR}$, to regulate macrophage apoptosis in the vessel wall. Strategies aimed at neutralizing MAARS expression or blocking MAARS-HuR interactions may provide a translational 
approach to limiting macrophage apoptosis and vascular remodeling in advanced plaques applicable to a range of chronic disease states.

\begin{abstract}
Methods
Cell culture and transfection. Bone marrow was isolated from the femur and tibia of C57BL/6 mice and cultured in IMDM medium supplemented with $10 \mathrm{ng} \mathrm{ml}^{-1}$ mMCSF (mouse macrophage colony stimulation factor (416 ML, R\&D), 10\% FBS, and $1 \%$ Penicilin-Streptomycin. Medium was changed every 2 days and cells were used in experiments after at 7-10 days in culture. Transfection was performed using Lipofectamine 3000 (Invitrogen, 11668-019) as described in manufacturer's protocol, and customized GapmeRs for MAARS (Qiagen, 25nmol except when mentioned differently) or negative control \#1 (Qiagen). Cells were allowed to grow for $36 \mathrm{~h}$ before treatment with CPT (Sigma-Milipore) or TNF-a (210-TA/CF, R\&D Systems) for 2 or $16 \mathrm{~h}$, respectively. Cells were lysed in TRIzol (Invitrogen) or RIPA buffer (Boston BioProdcuts, BP-115) for further qPCR and Western Blot analysis, respectively ${ }^{73,74}$
\end{abstract}

RNA isolation and RT-qPCR. Tissues were homogenized using TissueLyser II (Qiagen) according to manufacturer's instructions. For RNA isolation, TRIzol reagent (Invitrogen) or RNeasy kit (Qiagen) was used based on manufacturers protocol. Isolation of intimal RNA and subsequent RT-qPCR from aorta was performed as previously documented ${ }^{73,74}$. Briefly, aortas were carefully flushed with PBS, followed by intima peeling using TRIzol reagent (Invitrogen, 15596018). TRIzol was flushed for $10 \mathrm{~s}-10 \mathrm{~s}$ pause-another $10 \mathrm{~s}$ flushed and collected in an Eppendorf tube ( 300-400 $\mu \mathrm{l}$ total) and snap frozen in liquid nitrogen. The intima specific isolation was assessed by qPCR showing enrichment of endothelial marker CD31 and macrophage marker Mac2 in the intima fraction compared to media/ adventitia fraction (Supplementary Fig. 1c, d). Subsequent RT-qPCR was performed using High-Capacity cDNA Reverse Transcription kit (Applied Biosystems, 4368813). GoTaq qPCR Master Mix (Promega, A6001) was used for RT-qPCR experiments. Expression of mRNAs and lncRNA expression levels were normalized to GAPDH, HPRT, or $\beta$-actin (Aglient, AriaMx Real Time PCR System). Changes in expression were calculated using delta delta Ct method. Primer sequences are described in Table 2. MAARS copy number was quantified using the formula, as previously described ${ }^{75,76}$

$$
\text { number of copies }(\text { molecules })=\frac{X \mathrm{ng} \times 6.0221 \times 10^{23} \text { molecules } / \mathrm{mole}}{N \times 660 \mathrm{~g} / \mathrm{mole}^{\dagger} \times 1 \times 10^{9} \mathrm{ng} / \mathrm{g}},
$$

where Avogadro's number $=6.02 \times 10^{23}$ molecules $/ \mathrm{mole} ; X$ is the amount of amplicon (ng); $N$ is the length of dsDNA amplicon; $660 \mathrm{~g} / \mathrm{mole}=$ average mass of 1 bp RNA.

5' RACE-PCR. 5' RACE-PCR (Rapid amplification of cDNA ends-Polymerase Chain Reaction) was performed based on the manufacturer's protocol (ClonTech, 634858). Briefly, $0.5 \mu \mathrm{g}$ total RNA from mouse BMDM was used and combined with $1 \mu \mathrm{l}$ of gene-specific primer $(10 \mu \mathrm{M}$ stock) to a final volume of $11 \mu \mathrm{l}$. Tubes were incubated at $72{ }^{\circ} \mathrm{C}$ for $3 \mathrm{~min}$, followed by $68^{\circ} \mathrm{C}$ for $5 \mathrm{~min}$ and then placed immediately on ice. Protocol was followed as suggest by manufacturer. Primers were designed with $\mathrm{Tm}>70{ }^{\circ} \mathrm{C}$ (Table 2). Following touchdown PCR, the product was loaded on $1 \%$ agarose gel and bands were isolated and cloned in vector for sequencing.

Polyadenylation. RNA of $10^{6} \mathrm{BMDMs}$ was isolated using TRIzol reagent (Invitrogen) and resuspended in RNase-free water. Polyadenylated and nonpolyadenylated RNA was enriched with polyA Spin mRNA isolation kit (NEB, S1560S) based on manufacturer's protocol. RT-PCR was performed with same

Table 2 Mouse-specific primers.

Primers

MAARS forward

MAARS reverse

MAARS 5'RACE

HuR forward

HuR reverse

BCL2 forward

$B C L 2$ reverse

MCL1 forward

MCL1 reverse

Prothymosin A forward

Prothymosin A reverse

Caspase- 9 forward

Caspase -9 reverse

Caspase- 8 forward

Caspase- 8 reverse

Caspase-3 forward

Caspase-3 reverse

BAX forward

$B A X$ reverse

CDKN1B (p27) forward

CDKN1B (p27) reverse

p53 forward

p53 reverse

IL-1B forward

$\mathrm{IL}-1 \mathrm{~B}$ reverse

COX-2 forward

COX-2 reverse

TNFa forward

TNFa reverse

MCP-1 forward

MCP-1 reverse

Mac2 forward

Mac2 reverse

GAPDH forward

GAPDH reverse

U6 forward

U6 reverse

AREs synthetic RNA oligonucleotides sequence

TCACTTGTGCCCTGACTCTG

TCTCCAGCAĀ̄ACAATCCAG

GATTACGCCAAGCTTGGTAGGCTAAGGCTCAAAGCCTGGAACA

TTCTCGGTTTGGGCGAATCA

ACTTCACTGTGATGGGCTCG

GAACTGGGGGAGGATTGTGG

GCATGCTGGGGCCATATAGT

TGCTCCGGAAACTGGACATT

TCCTGCCCCAGTTTGTTACG

CTCTCGCCAGAGTCCTCGAA

GGAGCTGGTATCCACTGCC

TCCTGGTACATCGAGACCTTG

AAGTCCCTTTCGCAGAAACAG

TGCTTGGACTACATCCCACAC

TGCAGTCTAGGAAGTTGACCA

ATGGAGAACAACAAAACCTCAGT

TTGCTCCCATGTATGGTCTTTAC

TGAAGACAGGGGCCTTTTTG

AATTCGCCGGAGACACTCG

TCAAACGTGAGAGTGTCTAACG

CCGGGCCGAAGAGATTTCTG

GTCACAGCACATGACGGAGG

TCTTCCAGATGCTCGGGATAC

ATGCCACCTTTTGACAGTGATG

AGCTTCTCCACAGCCACAAT

TTCAACACACTCTATCACTGGC

AGAAGCGTTTGCGGTACTCAT

CCCTCACACTCAGATCATCTTCT

GCTACGACGTGGGCTACAG

TTAAAAACCTGGATCGGAACCAA

GCATTAGCTTCAGATTTACGGGT

GGAGAGGGAATGATGTTGCCT

TCCTGCTTCGTGTTACACACA

AGGTCGGTGTGAACGGATTTG

TGTAGACCATGTAGTTGAGGTCA

CTCGCTTCGGCAGCACA

AACGCTTCACGAATTTGCGT

UCAUUAUUUAUUACGAUUUAUUUAUUAGCGAUUUAUUUAUUUACG 
input volume, independent of concentration and normalized to nonpolyadenylated RNA fraction.

Cellular fractionation. BMDMs fractionation for cytoplasmic and nuclear fractions was performed using the Active Motif fractionation kit (40010, Active Motif) according to the manufacturer's protocol. The protein lysates concentration was determined and used for Western Blot. RNA was harvested as described previously and cleaned up using the RNeasy kit (Qiagen). Equivalent RNA volumes of cytoplasmic and nuclear associated RNA were converted to cDNA as described previously.

Western blot. Proteins were isolated using RIPA buffer (Boston BioProdcuts, BP$115)$ with protease inhibitor and phosphatase inhibitors. Protein concentrations were determined using Pierce BCA assay (Thermo Scientific). In total, $20 \mu \mathrm{g}$ protein were loaded per lane on a 4-20\% Mini-PROTEAN TGX Gel (Bio-Rad, 4561096). Separated proteins were transferred to PVDF membranes using the Transfer Turbo Blot system (Bio-Rad) and Trans-Blot Turbo RTA Transfer Kit (Bio-Rad, 170-4272). The membrane was blocked with 5\% nonfat milk in TBST for $1 \mathrm{~h}$ at room temperature. After blocking, the membrane was incubated overnight at $4{ }^{\circ} \mathrm{C}$ with antibodies against Flag Tag (Cell Signaling, 2368, 1:1000), GAPDH (Cell Signaling, 2118, 1:4000), $\beta$-actin (Cell Signaling, \#4970, 1:3000), cleaved Caspase-3 (Cell Signaling, 1:1000), cleaved Caspase-8 (Cell Signaling, 1:2000), HuR (12582S, Cell Signaling, 1:3000), Quantification of protein bands were performed using a luminescent image analyzer (Bio-Rad, Chemidoc).

Protein-coding potential. In silico CPAT online algorithm was used for prediction of coding potential ${ }^{77}$. Transcripts for MAARS (Ensemble ID\#

ENSMUST00000067618.4) were synthesized by Genewiz. For in vitro validation of peptide coding potential, MAARS transcript was cloned upstream of p3xFLAGCMV-14 expression vector (Sigma, E7908) using EcoRI restriction site. In total, $293 \mathrm{~T}$ cells were transfected with $500 \mathrm{ng}$ plasmid using Lipofectamine 2000 (Invitrogen) and protein lysate was isolated $72 \mathrm{~h}$ post-transfection, followed by immunoblotting for FLAG Tag (Cell Signaling, 8146).

RNA-ISH. Customized probe for MAARS was specifically developed to detect ENSMUST00000067618.4 (Advanced Cell Diagnostics). BMDMs were fixed in 4\% paraformaldehyde and the ISH protocol for cultured adherent cells was performed as described by the manufacturer (RNAscope 2.5 HD Reagent Kit-Red; Advanced Cell Diagnostics, 322350).

Apoptosis assays. TUNEL (Roche, 12156792910) and ApoLive-Glo Cleaved Caspase-3/7 (Promega G6410) assays were performed based on manufacturer's protocol. Briefly, BMDMs were seeded in 96-well plate (Corning, 3610) and transfected with gapmeRs. After $36 \mathrm{~h}$ the cells were activated with CPT $(20 \mu \mathrm{M})$ at indicated concentrations for $2 \mathrm{~h}$ and the reagents for each assay were added according to the protocols.

Efferocytosis. Efferocytosis assay was performed as described in ref. ${ }^{78}$. Briefly, $5 \times$ $10^{6}$ cells/ml Jurkat cells were labeled with $5 \mu \mathrm{M}$ AM Calcein (Invitrogen, LSH2452-50). After $2 \mathrm{~h}$ incubation, cells were washed and irradiated with UV $\left(150 \mathrm{~mJ} / \mathrm{cm}^{2}\right)$ with an open lid, followed by another $2 \mathrm{~h}$ incubation before apoptotic cells were added in a 1:1 ratio to gapmeR-transfected or lentivirus-transduced primary macrophages. After several rounds of gentle washing, macrophages were counted positive for internalized green apoptotic bodies if they contained $>3 \mu \mathrm{m}$ clusters of green dots. Quantification was performed from four images with a total of 400 macrophages. For quantification of in vivo efferocytosis, macrophages were stained using rat anti-Mac3 (BD Pharmingen, 553322, 1:900), as described below for immunofluorescence. TUNEL protocol was performed based on the manufacturer's protocol (Roche, In situ cell death detection kit, TMR red) and the ratio of macrophage-free TUNEL over macrophage-associated TUNEL signaling was calculated 79

Lentivirus production and transduction. Lentivirus for pUltra (Malcolm Moore, Addgene, 24129) were generated by co-transfection of 293 T cells (ATCC CRL3216) using Lipofectamine 3000 (Life Technologies) with pMD2.G (Didier Trono, Addgene, 12259) and psPAX2 (Didier Trono, Addgene, 12260) in a ratio 3:2:1, respectively. Transfection mix was added dropwise to dish and medium was changed after $8 \mathrm{~h}$. The supernatant was collected 2 days later by filtering through $0.45 \mu \mathrm{m}$ filter and stored at $-80^{\circ} \mathrm{C}$. Transduction of BMDM was carried out in 6well or 12-well plate adding $1 \mathrm{ml}$ lentiviral supernatant to $1 \mathrm{ml}$ medium in combination with $8 \mu \mathrm{g} / \mathrm{ml}$ polybrene (American Bio, AB01643). Normal growth medium was replaced after $36 \mathrm{~h}$

Cholesterol efflux determination. For cholesterol efflux determination, BMDMs were labeled with $2 \mu \mathrm{Ci} / \mathrm{ml}\left[1,2-{ }^{3} \mathrm{H}\right]$-cholesterol (Perkin Elmer, Waltham, MA, USA) for $24 \mathrm{~h}$ in the presence of an acyl-CoA:cholesterol acyltransferase inhibitor (Sandoz 58035, $2 \mu \mathrm{g} / \mathrm{ml}$; Sigma-Aldrich, Milano, Italy) and $25 \mu \mathrm{g} / \mathrm{ml}$ acetylated
LDL isolated and chemically modified from healthy donors by ultracentrifugation $^{80,81}$. BMDMs were then incubated with $0.2 \%$ free fatty acid bovine serum albumin (BSA; Sigma-Aldrich, Milano, Italy) in IMDM for $18 \mathrm{~h}$ and then treated for $6 \mathrm{~h}$ with $10 \mu \mathrm{g} / \mathrm{ml}$ lipid free human apolipoprotein AI (Sigma-Aldrich, Milano, Italy), $12,5 \mu \mathrm{g} / \mathrm{ml} \mathrm{HDL}$ (isolated from healthy donors by ultracentrifugation ${ }^{81}$, or $2 \%(\mathrm{v} / \mathrm{v})$ human serum (NS) obtained from a pool of normolipidemic subjects, as cholesterol acceptors. Cholesterol efflux was expressed as a percentage of $\left[1,2-{ }^{3} \mathrm{H}\right]$ cholesterol released into the medium over the total radioactivity incorporated by cells.

LncRNA pulldown and liquid chromatography-mass spectrometry (LC-MS/MS). LncRNA pulldown and LC-MS/MS was performed as previously documente ${ }^{45}$. For lncRNA pulldown, biotinylated RNA was generated using T7 RNA polymerase (Promega, P1300) by adding $1 \mu \mathrm{g}$ linearized plasmid DNA, Biotin RNA labeling mix (Roche, 11685597910), 10× transcription buffer (Promega, P1300) and related RNase-free water in a total volume of $20 \mu \mathrm{l}$. The mix was incubated at $37^{\circ} \mathrm{C}$ for $2 \mathrm{~h}$ After incubation, $2 \mu \mathrm{l}$ Dnase I (NEB, M0303L) was added and incubated at $37^{\circ} \mathrm{C}$ for another $15 \mathrm{~min}$ to remove DNA template and the reaction was stopped by adding $0.8 \mu \mathrm{l} 0.5 \mathrm{M}$ EDTA ( $\mathrm{pH}$ 8.0). Biotinylated RNA purification was performed using $\mathrm{G}$ 50 Sephadex Columns (Roche, 11273965001) according to the manufacturer's protocol. After determination of the RNA concentration, the purified biotinylated RNA was immediately used or stored at $-80^{\circ} \mathrm{C}$. Ten pmol of biotinylated RNA was heated for $2 \mathrm{~min}$ at $90^{\circ} \mathrm{C}$ in RNA structure buffer $(10 \mathrm{mM}$ Tris $\mathrm{pH} 7.0,0.1 \mathrm{M} \mathrm{KCl}, 10 \mathrm{mM}$ $\mathrm{MgCl} 2$ ). After incubation, immediately transfer the mix on ice and incubate for another $2 \mathrm{~min}$ and incubated at room temperature for $20 \mathrm{~min}$. Nuclear pellets were prepared by resuspending pellet of 107 HUVECs in $2 \mathrm{ml}$ ice-cold PBS, $2 \mathrm{ml}$ ice-cold unclear isolation buffer (1.28 M sucrose, $40 \mathrm{mM}$ Tris- $\mathrm{HCl} \mathrm{pH} 7.4,20 \mathrm{mM} \mathrm{MgCl} 2,4 \%$ Triton X-100) and $6 \mathrm{ml}$ ice-cold RNase-free water and incubated on ice for $20 \mathrm{~min}$. After incubation, nuclei pellets were harvested by centrifugation at $2500 \times g$ for 15 min, nuclear pellets were then resuspended in $1 \mathrm{ml} \mathrm{RIP} \mathrm{buffer}(0.15 \mathrm{M} \mathrm{KCl}, 25 \mathrm{mM}$ Tris- $\mathrm{HCl}$ pH 7.4, 5 mM EDTA, 0.5\% NP-40, 0.5 mM DTT (Sigma, 646563), $100 \mathrm{U} /$ $\mathrm{ml}$ RNase inhibitor (Invitrogen, AM2684), 1x protease inhibitor cocktail). Nuclei were homogenized by 18 strokes using a dounce homogenizer, followed by centrifuging for $15 \mathrm{~min}$ at $15,000 \times g$. The supernatant containing nuclear protein was transferred to a new tube and precleared by applying $60 \mu \mathrm{l}$ of Streptavidin agarose beads (Thermo Scientific, 20347) for $1 \mathrm{~h}$ at $4{ }^{\circ} \mathrm{C}$. Ten pmol properly folded biotinylated RNA and $1 \mu \mathrm{g} / \mu \mathrm{l}$ yeast tRNA (Ambion, AM7119) were added into the precleared nuclear lysate $(200 \mu \mathrm{g})$ and incubated for $2 \mathrm{~h}$ at $4{ }^{\circ} \mathrm{C}$, followed by addition of $60 \mu$ of Steptavidin agarose beads and incubation for $1 \mathrm{~h}$ at $4^{\circ} \mathrm{C}$. At the end of the incubation, beads were collected by centrifugation at $12,000 \times g$ and washed with $1 \mathrm{ml}$ ice-cold NT2 buffer ( $50 \mathrm{mM}$ Tris- $\mathrm{HCl}$ pH 7.4, $0.15 \mathrm{M} \mathrm{NaCl}, 1 \mathrm{mM} \mathrm{MgCl} 2,0.05 \%$ NP-40, $100 \mathrm{U} / \mathrm{ml}$ RNase inhibitor, $400 \mathrm{nM}$ Vanadyl-ribonucleoside complex (BioLabs, S1402S), 1x protease inhibitor cocktail) at $4{ }^{\circ} \mathrm{C}$ five times. After washing, proteins were denaturated in $40 \mu \mathrm{l} 2 \times$ Laemmli loading buffer (4\%SDS, $120 \mathrm{mM}$ Tris$\mathrm{HCl} \mathrm{pH} 6.8,0.02 \%$ bromophenol blue, $0.2 \mathrm{M} \mathrm{DTT}$ ) at $98^{\circ} \mathrm{C}$ for $8 \mathrm{~min}$ for subsequent immunoblotting or resuspended in wash buffer without NP-40 for mass spectrometry analysis. For in vivo pulldown, biotinylated RNA was injected on two constitutive days by tail vein $(15 \mu \mathrm{g} /$ injection) before aortas were isolated on day 3 . The tissue was processed as described above for cell lysate.

\section{Liquid chromatography-mass spectrometry (LC-MS/MS). LC-MS/MS was} performed as previously described ${ }^{82}$. Briefly, IncRNA pulldown of MAARS or LacZ purified samples were reduced with $10 \mathrm{mM}$ DTT for $30 \mathrm{~min}$ at $56^{\circ} \mathrm{C}$ in the presence of $0.1 \%$ RapiGest SF (Waters). Cysteines were alkylated with $22.5 \mathrm{mM}$ iodoacetamide for $20 \mathrm{~min}$ at room temperature in the dark. Samples were digested overnight at $37^{\circ} \mathrm{C}$ with trypsin. Rapigest was then cleaved according to manufacturer's instructions and peptides purified by reversed phase and strong cation exchange chromatography. Peptides were loaded onto a precolumn $(4 \mathrm{~cm}$ POROS 10R2, Applied Biosystems), resolved on a self-packed analytical column $(12 \mathrm{~cm}$ Monitor C18, Column Engineering) after gradient elution (NanoAcquity UPLC system, Waters; $5-35 \% \mathrm{~B}$ in $90 \mathrm{~min} ; A=0.2 \mathrm{M}$ acetic acid in water, $B=0.2 \mathrm{M}$ acetic acid in acetonitrile), and introduced to the MS (TripleTOF 5600, ABSciex, Framingham, MA) by ESI (spray voltage $=2.2 \mathrm{kV}$ ). The mass spectrometer was programmed to perform data-dependent MS/MS (unit resolution, $m / z 100-2000$ ) on the 20 most abundant precursors in each MS1 scan $(\mathrm{m} / z$ 300-2000; accumulation time $=0.5 \mathrm{~s}$; threshold $=70$ counts; charge state $=2+$ to $5+$ ) using a rolling collision energy. After MS/MS, each precursor was excluded for $25 \mathrm{~s}$. Raw data were converted to.mgf using ABSciex MSDataConverter; precursor and product ions were recalibrated using a linear equation derived from fitting experimentally observed masses obtained in an initial low mass tolerance database search. Recalibrated data were matched to peptide sequences in a forward/reversed human NCBI refseq database using Mascot version 2.4.1. Search parameters included trypsin specificity with up to two missed cleavages, fixed carbamidomethylation $(\mathrm{C},+57 \mathrm{Da})$ and variable oxidation $(\mathrm{M},+16 \mathrm{Da})$. Precursor and product mass tolerances were $12 \mathrm{ppm}$ and $25 \mathrm{mmu}$. Protein hits from MAARS-specific pulldown were compared to negative control ( $\mathrm{LacZ})(n=2$, with two technical replicates).

Animal studies. All protocols concerning animal use were approved by the Institutional Animal Care and Use Committee at Brigham and Women's Hospital 
and Harvard Medical School, Boston, MA and conducted in accordance with the National Institutes of Health Guide for the Care and Use of Laboratory Animals. Studies were performed in $\mathrm{LDLR}^{-1-}$ mice (Jackson Laboratory, Stock\#: 002207) or in $\mathrm{C} 57 \mathrm{Bl} / 6$ mice (Charles River, Strain code\#027).

Immunohistology and characterization of atherosclerotic lesions. To quantify atherosclerosis in $\mathrm{LDLR}^{-1-}$ mice that were placed on HCD (Research Diets Inc., D12108C), aortic roots and aortic arch were embedded in OCT and frozen at $-80^{\circ} \mathrm{C}$. Serial cryostat sections $(6 \mu \mathrm{m})$ were prepared using tissue processor Leica $\mathrm{CM} 3050$. For lesion characterizations, the thoracic-abdominal aorta and aortic root were stained for ORO, macrophages (anti-Mac3, BD Pharmingen, 553322, 1:900) T cells (anti-CD4, BD Pharmingen, 553043, 1:90; anti-CD8, Chemicon, CBL1318, 1:100), MHC-positive cells (anti-MHCII, Novus Biologicals), and vascular smooth muscle cells (SM- $\alpha$-actin, Sigma, F-3777, 1:500) ${ }^{73,83}$. The staining area was measured using Image-Pro Plus software, Media Cybernetics, and CD4+ and CD8+ cells were counted manually.

Immunofluorescence staining. For immunofluorescence staining, $6 \mu \mathrm{m}$ frozen sections of aortic roots and aortic arches were fixed with cold-acetone or $4 \%$ paraformaldehyde for 5 and $15 \mathrm{~min}$, respectively, and blocked in PBS containing $3 \% \mathrm{BSA}$ for $1 \mathrm{~h}$ at room temperature. Sections were stained with rat anti-Mac2, (Cedarlane, CL8942AP, 1:100), anti-Mac3 (BD Pharmingen, 553322, 1:900), rabbit anti-cleaved caspase-3 (Cell Signaling, 9664, 1:400) rabbit anti-HuR/ELAVL1 (12582S Cell Signaling and AB242410, Abcam), biotin goat anti-MerTK (BAF591, R\&D Technologies) in PBS at $4{ }^{\circ} \mathrm{C}$ overnight. Similarly, BMDMs were plated on microscope slides and after treatment were fixed in $4 \%$ paraformaldehyde, permeabilized with $0.1 \%$ Triton X-100, and incubated with selected antibodies overnight as above. After three washes with PBS, the sections were further incubated with Alexa Flour 488-conjugated donkey anti-Rat IgG (Invitrogen, A21208, 1:300) and Alexa Flour 555-conjugated goat anti-Rabbit IgG (Invitrogen, A21428, 1:300) and anti-streptavidin Alexa-488 (Life Technologies) in PBS for $1 \mathrm{~h}$ at room temperature. Nuclei were stained for $5 \mathrm{~min}$ at room temperature in PBS containing DAPI (Cell signaling, 4083, $0.5 \mu \mathrm{g} / \mathrm{ml}$ ). Coverslips were mounted with ProLong Gold antifade reagent (Invitrogen, P36930). Images were acquired on an upright Carl Zeiss LSM 510 confocal. TUNEL protocol was performed as described by manufacturer's protocol (In Situ Cell Death Detection Kit TMR red, 12156792910, Roche).

Lipid profile analysis. Lipid profile was measured as follows: the triglycerides were determined using InfinityTM Triglycerides Liquid Stable Reagent (Thermo Scientific). Total cholesterol was measured using the InfinityTM Cholesterol Reagent (Thermo Scientific) and HDL cholesterol was measured by colorimetric assay (BioAssay Systems, EnzyChromTM HDL). LDL cholesterol levels were calculated using the following formula: $\mathrm{LDL}=$ total cholesterol $-\mathrm{HDL}$ cholesterol - (triglycerides $/ 5)^{73}$. Standards were purchased from Pointe Scientific, Inc.

Liver function assays. AST (cat. No. EASTR-100) and ALT (cat. No. EALT-100) were detected using the BioAssay Systems kits according to the manufacturer's protocol.

RNA-Seq analysis. RNA-Seq analysis was performed after ribodepletion and standard library construction using Illumina HiSeq2500 V4 2×100 PE (Genewiz, South Plainfield, NJ). All samples were processed using an RNA-seq pipeline implemented in the bcbio-nextgen project (https://bcbio-nextgen.readthedocs.org/ en/latest/ $)^{17}$. Raw reads were examined for quality issues using FastQC (http:// www.bioinformatics.babraham.ac.uk/projects/fastqc/) to ensure library generation and sequencing were suitable for further analysis. Trimmed reads were aligned to UCSC build mm10 of the Mouse genome, augmented with transcript information from Ensembl release 79 using STAR ${ }^{84}$. Alignments were checked for evenness of coverage, rRNA content, genomic context of alignments (for example, alignments in known transcripts and introns), complexity, and other quality checks using a combination of FastQC, Qualimap. Counts of reads aligning to known genes were generated by featureCounts ${ }^{85}$. Differential expression at the gene level were called with DESeq2. The total gene hit counts and CPM values were calculated for each gene and for downstream differential expression analysis between specified groups was performed using DESeq2 and an adapted DESeq2 algorithm, which excludes overlapping reads, called no-overlapping reads. Genes with adjusted FDR $<0.05$ and $\log 2$-fold change (1.5) were called as differentially expressed genes for each comparison. Mean quality score of all samples was 35.67 within a range of $40,000,000-50,000,000$ reads per sample. All samples had at least $>70 \%$ of mapped fragments over total. "MetaCore (v20.2) was used for functional enrichment analysis."

Statistics. Data throughout the paper are expressed as mean \pm SD. Statistical differences were calculated using unpaired two-tailed Student's $t$ test or one-way ANOVA with Bonferroni correction for multiple comparisons. A probability of $p<$ 0.05 was considered statistically significant. Ns not significant; ${ }^{*} p<0.05 ;{ }^{* *} p<0.01$;
${ }^{* * *} p<0.001 ; * * * * p<0.0001$. For illustration of differentially expressed genes Prism GraphPad software (V.7.0a) was used.

Reporting summary. Further information on research design is available in the Nature Research Reporting Summary linked to this article.

\section{Data availability}

All relevant data are available from the authors. The RNA-seq data are accessible at: https://www.ncbi.nlm.nih.gov/geo/query/acc.cgi?acc=GSE155842. Source data are provided with this paper.

Received: 14 December 2019; Accepted: 26 October 2020; Published online: 01 December 2020

\section{References}

1. Libby, P., Ridker, P. M. \& Hansson, G. K. Progress and challenges in translating the biology of atherosclerosis. Nature 473, 317-325 (2011).

2. Libby, P. Inflammation in atherosclerosis. Arterioscler. Thromb. Vasc. Biol. 32, 2045-2051 (2012)

3. Thorp, E., Subramanian, M. \& Tabas, I. The role of macrophages and dendritic cells in the clearance of apoptotic cells in advanced atherosclerosis. Eur. J. Immunol. 41, 2515-2518 (2011).

4. Li, Y. et al. Cholesterol-induced apoptotic macrophages elicit an inflammatory response in phagocytes, which is partially attenuated by the Mer receptor. J. Biol. Chem. 281, 6707-6717 (2006).

5. Kavurma, M. M., Rayner, K. J. \& Karunakaran, D. The walking dead: macrophage inflammation and death in atherosclerosis. Curr. Opin. Lipido. 28, 91-98 (2017).

6. Rayner, K. J. Cell death in the vessel wall: the good, the bad, the ugly. Arterioscler. Thromb. Vasc. Biol. 37, e75-e81 (2017).

7. Okazaki, Y. et al. Analysis of the mouse transcriptome based on functional annotation of 60,770 full-length cDNAs. Nature 420, 563-573 (2002).

8. Kapranov, P. et al. RNA maps reveal new RNA classes and a possible function for pervasive transcription. Science 316, 1484-1488 (2007).

9. Haemmig, S., Simion, V. \& Feinberg, M. W. Long Non-Coding RNAs in Vascular Inflammation. Front. Cardiovasc. Med. 5, 22 (2018).

10. Simion, V., Haemmig, S. \& Feinberg, M. W. LncRNAs in vascular biology and disease. Vasc. Pharm. 114, 145-156 (2019).

11. Bell, R. D. et al. Identification and initial functional characterization of a human vascular cell-enriched long noncoding RNA. Arterioscler. Thromb. Vasc. Biol. 34, 1249-1259 (2014).

12. Abdelmohsen, K., Lal, A., Kim, H. H. \& Gorospe, M. Posttranscriptional orchestration of an anti-apoptotic program by HuR. Cell Cycle 6, 1288-1292 (2007).

13. Brennan, C. M. \& Steitz, J. A. HuR and mRNA stability. Cell Mol. Life Sci. 58 266-277 (2001).

14. Gorospe, M. HuR in the mammalian genotoxic response: post-transcriptional multitasking. Cell Cycle 2, 412-414 (2003).

15. von Roretz, C. et al. Apoptotic-induced cleavage shifts HuR from being a promoter of survival to an activator of caspase-mediated apoptosis. Cell Death Differ. 20, 154-168 (2013)

16. Mazan-Mamczarz, K. et al. RNA-binding protein HuR enhances p53 translation in response to ultraviolet light irradiation. Proc. Natl Acad. Sci. USA 100, 8354-8359 (2003).

17. Haemmig, S. et al. Long noncoding RNA SNHG12 integrates a DNA-PKmediated DNA damage response and vascular senescence. Sci. Transl. Med. 12. https://doi.org/10.1126/scitranslmed.aaw1868 (2020).

18. Shen, W. et al. Chemical modification of PS-ASO therapeutics reduces cellular protein-binding and improves the therapeutic index. Nat. Biotechnol. 37, 640-650 (2019)

19. Lee, J. E. et al. Fermentation of green tea with $2 \%$ aquilariae lignum increases the anti-diabetic activity of green tea aqueous extracts in the high fat-fed mouse. Nutrients 7, 9046-9078 (2015).

20. Kim, J. et al. The angiogenesis inhibitor ALS-L1023 from lemon-balm leaves attenuates high-fat diet-induced nonalcoholic fatty liver disease through regulating the visceral adipose-tissue function. Int. J. Mol. Sci. 18. https://doi. org/10.3390/ijms18040846 (2017)

21. Seimon, T. \& Tabas, I. Mechanisms and consequences of macrophage apoptosis in atherosclerosis. J. Lipid Res. 50, S382-S387 (2009).

22. Yoshida, A., Ueda, T., Wano, Y. \& Nakamura, T. DNA damage and cell killing by camptothecin and its derivative in human leukemia HL-60 cells. Jpn J. Cancer Res. 84, 566-573 (1993).

23. Bhandare, S., Goldberg, D. S. \& Dowell, R. Discriminating between HuR and TTP binding sites using the k-spectrum kernel method. PLOS ONE 12, e0174052 (2017). 
24. Lebedeva, S. et al. Transcriptome-wide analysis of regulatory interactions of the RNA-binding protein HuR. Mol. Cell 43, 340-352 (2011).

25. Dan, C. et al. Modulation of TNF-alpha mRNA stability by human antigen R and miR181s in sepsis-induced immunoparalysis. EMBO Mol. Med 7, 140-157 (2015).

26. Mukherjee, N. et al. Integrative regulatory mapping indicates that the RNAbinding protein HuR couples pre-mRNA processing and mRNA stability. Mol. Cell 43, 327-339 (2011).

27. Doller, A. et al. Protein kinase $\mathrm{C}$ alpha-dependent phosphorylation of the mRNA-stabilizing factor HuR: implications for posttranscriptional regulation of cyclooxygenase-2. Mol. Biol. Cell 18, 2137-2148 (2007).

28. Lu, S., Mott, J. L. \& Harrison-Findik, D. D. Saturated fatty acids induce posttranscriptional regulation of HAMP mRNA via AU-rich element-binding protein, human antigen R (HuR). J. Biol. Chem. 290, 24178-24189 (2015).

29. Badawi, A. et al. Inhibition of IRES-dependent translation of caspase-2 by HuR confers chemotherapeutic drug resistance in colon carcinoma cells. Oncotarget 9, 18367-18385 (2018).

30. Hsiao, Y. W. et al. CCAAT/enhancer binding protein delta in macrophages contributes to immunosuppression and inhibits phagocytosis in nasopharyngeal carcinoma. Sci. Signal 6, ra59 (2013).

31. Van Vre, E. A., Ait-Oufella, H., Tedgui, A. \& Mallat, Z. Apoptotic cell death and efferocytosis in atherosclerosis. Arterioscler. Thromb. Vasc. Biol. 32, 887-893 (2012).

32. Thorp, E., Cui, D., Schrijvers, D. M., Kuriakose, G. \& Tabas, I. Mertk receptor mutation reduces efferocytosis efficiency and promotes apoptotic cell accumulation and plaque necrosis in atherosclerotic lesions of apoe $-/-$ mice. Arterioscler. Thromb. Vasc. Biol. 28, 1421-1428 (2008).

33. Wang, W. et al. Macrophage inflammation, erythrophagocytosis, and accelerated atherosclerosis in Jak2 (V617F) mice. Circ. Res. 123, e35-e47 (2018).

34. Kockx, M. M. et al. Apoptosis and related proteins in different stages of human atherosclerotic plaques. Circulation 97, 2307-2315 (1998).

35. Kockx, M. M. \& Herman, A. G. Apoptosis in atherosclerosis: beneficial or detrimental? Cardiovasc. Res. 45, 736-746 (2000).

36. van Vlijmen, B. J. et al. Macrophage p53 deficiency leads to enhanced atherosclerosis in $\mathrm{APOE}^{*} 3$-Leiden transgenic mice. Circ. Res. 88, 780-786 (2001).

37. Liu, J. et al. Reduced macrophage apoptosis is associated with accelerated atherosclerosis in low-density lipoprotein receptor-null mice. Arterioscler. Thromb. Vasc. Biol. 25, 174-179 (2005).

38. Arai, S. et al. A role for the apoptosis inhibitory factor AIM/Spalpha/Api6 in atherosclerosis development. Cell Metab. 1, 201-213 (2005).

39. Feng, B. et al. Niemann-Pick C heterozygosity confers resistance to lesional necrosis and macrophage apoptosis in murine atherosclerosis. Proc. Natl Acad. Sci. USA 100, 10423-10428 (2003).

40. Han, S. et al. Macrophage insulin receptor deficiency increases ER stressinduced apoptosis and necrotic core formation in advanced atherosclerotic lesions. Cell Metab. 3, 257-266 (2006).

41. Viereck, J. et al. Long noncoding RNA Chast promotes cardiac remodeling. Sci. Transl. Med. 8, 326ra322 (2016).

42. Leucci, E. et al. Melanoma addiction to the long non-coding RNA SAMMSON. Nature 531, 518-522 (2016).

43. Minamino, T. \& Komuro, I. Vascular cell senescence: contribution to atherosclerosis. Circ. Res. 100, 15-26 (2007).

44. Sun, X., Haider Ali, M. S. S. \& Moran, M. The role of interactions of long noncoding RNAs and heterogeneous nuclear ribonucleoproteins in regulating cellular functions. Biochem. J. 474, 2925-2935 (2017).

45. Feng, Y. et al. Methods for the study of long noncoding RNA in cancer cell signaling. Methods Mol. Biol. 1165, 115-143 (2014).

46. Torres, M. et al. RNA pull-down procedure to identify RNA targets of a long non-coding RNA. J. Vis. Exp. https://doi.org/10.3791/57379 (2018).

47. Haemmig, S., Simion, V., Yang, D., Deng, Y. \& Feinberg, M. W. Long noncoding RNAs in cardiovascular disease, diagnosis, and therapy. Curr. Opin. Cardiol. 32, 776-783 (2017).

48. Talwar, S. et al. Caspase-mediated cleavage of RNA-binding protein HuR regulates c-Myc protein expression after hypoxic stress. J. Biol. Chem. 286, 32333-32343 (2011).

49. Abdelmohsen, K. et al. Phosphorylation of HuR by Chk2 regulates SIRT1 expression. Mol. Cell 25, 543-557 (2007).

50. Wang, W., Caldwell, M. C., Lin, S., Furneaux, H. \& Gorospe, M. HuR regulates cyclin A and cyclin B1 mRNA stability during cell proliferation. ЕMBO J. 19, 2340-2350 (2000).

51. Kullmann, M., Gopfert, U., Siewe, B. \& Hengst, L. ELAV/Hu proteins inhibit p27 translation via an IRES element in the p27 5'UTR. Genes Dev. 16, 3087-3099 (2002).

52. Izquierdo, J. M. Hu antigen $\mathrm{R}(\mathrm{HuR})$ functions as an alternative pre-mRNA splicing regulator of Fas apoptosis-promoting receptor on exon definition. J. Biol. Chem. 283, 19077-19084 (2008).
53. von Roretz, C. \& Gallouzi, I. E. Protein kinase RNA/FADD/caspase-8 pathway mediates the proapoptotic activity of the RNA-binding protein human antigen R (HuR). J. Biol. Chem. 285, 16806-16813 (2010).

54. Filippova, N. et al. The RNA-binding protein HuR promotes glioma growth and treatment resistance. Mol. Cancer Res. 9, 648-659 (2011).

55. Lal, A., Kawai, T., Yang, X., Mazan-Mamczarz, K. \& Gorospe, M. Antiapoptotic function of RNA-binding protein HuR effected through prothymosin alpha. EMBO J. 24, 1852-1862 (2005).

56. Muralidharan, R. et al. Tumor-targeted nanoparticle delivery of HuR siRNA inhibits lung tumor growth in vitro and in vivo by disrupting the oncogenic activity of the RNA-binding protein HuR. Mol. Cancer Ther. 16, 1470-1486 (2017).

57. Katsanou, V. et al. The RNA-binding protein Elavll/HuR is essential for placental branching morphogenesis and embryonic development. Mol. Cell Biol. 29, 2762-2776 (2009).

58. Mazroui, R. et al. Caspase-mediated cleavage of HuR in the cytoplasm contributes to pp32/PHAP-I regulation of apoptosis. J. Cell Biol. 180, 113-127 (2008).

59. Zhou, H. L., Geng, C., Luo, G. \& Lou, H. The p97-UBXD8 complex destabilizes mRNA by promoting release of ubiquitinated HuR from mRNP. Genes Dev. 27, 1046-1058 (2013).

60. $\mathrm{Du}, \mathrm{M}$. et al. The LPS-inducible lncRNA Mirt2 is a negative regulator of inflammation. Nat. Commun. 8, 2049 (2017).

61. Yurdagul, A. Jr., Doran, A. C., Cai, B., Fredman, G. \& Tabas, I. A. Mechanisms and consequences of defective efferocytosis in atherosclerosis. Front. Cardiovasc. Med. 4, 86 (2017).

62. Bhatia, V. K. et al. Complement C1q reduces early atherosclerosis in lowdensity lipoprotein receptor-deficient mice. Am. J. Pathol. 170, 416-426 (2007).

63. McCubbrey, A. L. et al. MicroRNA-34a negatively regulates efferocytosis by tissue macrophages in part via SIRT1. J. Immunol. 196, 1366-1375 (2016).

64. Liu, B., Zhang, B., Guo, R., Li, S. \& Xu, Y. Enhancement in efferocytosis of oxidized low-density lipoprotein-induced apoptotic RAW264.7 cells through Sirt1-mediated autophagy. Int. J. Mol. Med. 33, 523-533 (2014).

65. Imperatore, F. et al. SIRT1 regulates macrophage self-renewal. EMBO J. 36, 2353-2372 (2017).

66. Park, S. Y. et al. SIRT1/adenosine monophosphate-activated protein kinase alpha signaling enhances macrophage polarization to an anti-inflammatory phenotype in rheumatoid arthritis. Front. Immunol. 8, 1135 (2017).

67. Zhang, Z. et al. Mouse macrophage specific knockout of SIRT1 influences macrophage polarization and promotes angiotensin II-induced abdominal aortic aneurysm formation. J. Genet. Genomics 45, 25-32 (2018).

68. Qin, C., Nagao, T., Grosheva, I., Maxfield, F. R. \& Pierini, L. M. Elevated plasma membrane cholesterol content alters macrophage signaling and function. Arterioscler. Thromb. Vasc. Biol. 26, 372-378 (2006).

69. Adorni, M. P., Ronda, N., Bernini, F. \& Favari, E. Rac1 and cholesterol metabolism in macrophage. J. Cardiovasc. Pharm. 62, 418-424 (2013).

70. Devries-Seimon, T. et al. Cholesterol-induced macrophage apoptosis requires ER stress pathways and engagement of the type A scavenger receptor. J. Cell Biol. 171, 61-73 (2005).

71. Yvan-Charvet, L. et al. ABCA1 and ABCG1 protect against oxidative stressinduced macrophage apoptosis during efferocytosis. Circ. Res. 106, 1861-1869 (2010).

72. Tabas, I. Apoptosis and plaque destabilization in atherosclerosis: the role of macrophage apoptosis induced by cholesterol. Cell Death Differ. 11, S12-S16 (2004).

73. Sun, X. et al. Systemic delivery of microRNA-181b inhibits nuclear factorkappaB activation, vascular inflammation, and atherosclerosis in apolipoprotein E-deficient mice. Circ. Res. 114, 32-40 (2014).

74. Sun, X. et al. MicroRNA-181b regulates NF-kappaB-mediated vascular inflammation. J. Clin. Invest. 122, 1973-1990 (2012).

75. Joshi, M., Keith Pittman, H., Haisch, C. \& Verbanac, K. Real-time PCR to determine transgene copy number and to quantitate the biolocalization of adoptively transferred cells from EGFP-transgenic mice. Biotechniques 45, 247-258 (2008).

76. Cheng, H. S. et al. MicroRNA-146 represses endothelial activation by inhibiting pro-inflammatory pathways. EMBO Mol. Med. 5, 1017-1034 (2013).

77. Wang, L. et al. CPAT: coding-potential assessment tool using an alignmentfree logistic regression model. Nucleic Acids Res. 41, e74 (2013).

78. Thorp, E. et al. Reduced apoptosis and plaque necrosis in advanced atherosclerotic lesions of Apoe- $-/-$ and Ldlr-/- mice lacking CHOP. Cell Metab. 9, 474-481 (2009).

79. Wang, Y. et al. Mitochondrial fission promotes the continued clearance of apoptotic cells by macrophages. Cell 171, 331-345.e322 (2017).

80. Havel, R. J., Eder, H. A. \& Bragdon, J. H. The distribution and chemical composition of ultracentrifugally separated lipoproteins in human serum. J. Clin. Invest. 34, 1345-1353 (1955). 
81. Basu, S. K., Goldstein, J. L., Anderson, G. W. \& Brown, M. S. Degradation of cationized low density lipoprotein and regulation of cholesterol metabolism in homozygous familial hypercholesterolemia fibroblasts. Proc. Natl Acad. Sci. USA 73, 3178-3182 (1976).

82. Ficarro, S. B. et al. Improved electrospray ionization efficiency compensates for diminished chromatographic resolution and enables proteomics analysis of tyrosine signaling in embryonic stem cells. Anal. Chem. 81, 3440-3447 (2009).

83. Cao, Z. et al. Kruppel-like factor KLF10 targets transforming growth factorbetal to regulate CD4(+)CD25(-) T cells and T regulatory cells. J. Biol. Chem. 284, 24914-24924 (2009).

84. Dobin, A. et al. STAR: ultrafast universal RNA-seq aligner. Bioinformatics 29, 15-21 (2013).

85. Liao, Y., Smyth, G. K. \& Shi, W. featureCounts: an efficient general purpose program for assigning sequence reads to genomic features. Bioinformatics $\mathbf{3 0}$, 923-930 (2014).

\section{Acknowledgements}

The authors would like to thank Ana Lay-Hong and Aniket P. Gad for their assistance with immunofluorescence imaging (Harvard Digestive Disease Center, NIH P30DK034854). This work was supported by the National Institutes of Health (HL115141, HL134849, HL148207, HL148355, HL153356 to M.W.F.), the American Heart Association (18SFRN33900144 and 20SFRN35200163 to M.W.F., 18POST34030395 to S.H.), the Arthur K. Watson Charitable Trust (to M.W.F.), and the Dr. Ralph and Marian Falk Medical Research Trust (to M.W.F.), a Sarnoff Cardiovascular Research Foundation Fellowship (to J.B.P.), and the Swiss National Science Foundation (P2BEP3_162063 to S.H.).

\section{Author contributions}

Conceived the hypothesis: M.W.F. and V.S.; performed the experiments: V.S., H.Z., J.B.P., S.M., Y.T., J.F.L., B.P., and J.A.M.; designed and interpreted the results: V.S., H.Z., J.B.P.,

S.H., D.P.-C., N.R., B.P., J.A.M., A.F.C., and M.W.F.; wrote the manuscript: V.S. and M.W.F.

\section{Competing interests}

The authors declare no competing interests.

\section{Additional information}

Supplementary information is available for this paper at https://doi.org/10.1038/s41467020-19664-2.

Correspondence and requests for materials should be addressed to M.W.F.

Peer review information Nature Communications thanks the anonymous reviewer(s) for their contribution to the peer review of this work.

Reprints and permission information is available at http://www.nature.com/reprints

Publisher's note Springer Nature remains neutral with regard to jurisdictional claims in published maps and institutional affiliations.

(c) Open Access This article is licensed under a Creative Commons Attribution 4.0 International License, which permits use, sharing, adaptation, distribution and reproduction in any medium or format, as long as you give appropriate credit to the original author(s) and the source, provide a link to the Creative Commons license, and indicate if changes were made. The images or other third party material in this article are included in the article's Creative Commons license, unless indicated otherwise in a credit line to the material. If material is not included in the article's Creative Commons license and your intended use is not permitted by statutory regulation or exceeds the permitted use, you will need to obtain permission directly from the copyright holder. To view a copy of this license, visit http://creativecommons.org/ licenses/by/4.0/.

(C) The Author(s) 2020 